Fraszczyk A, Lamb T, Marinov $M$.

Are railways really that bad? An evaluation of rail systems

performance in Europe.

Transportation Research Part A 2016, 94, 573-591.

\section{Copyright:}

(C) 2016. This manuscript version is made available under the CC-BY-NC-ND 4.0 license

DOI link to article:

http://dx.doi.org/10.1016/i.tra.2016.10.018

Date deposited:

$16 / 11 / 2016$

Embargo release date:

03 May 2018

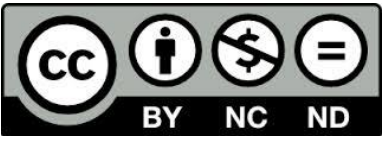

This work is licensed under a

Creative Commons Attribution-NonCommercial-NoDerivatives 4.0 International licence 


\title{
Are railways really that bad? An evaluation of rail systems performance in Europe with a focus on passenger rail
}

\author{
Anna Fraszczyk ${ }^{\mathrm{a}}$, Thomas Lamb ${ }^{\mathrm{b}}$, Marin Marinov ${ }^{\mathrm{a}}$ \\ a: NewRail Newcastle Centre for Railway Research, Newcastle University, Newcastle upon Tyne, NE1 7RU, United Kingdom; E-Mails: \\ anna.fraszczyk@newcastle.ac.uk; marin.marinov@newcastle.ac.uk \\ b: St Aidan's Catholic Academy, Willowbank Road, Ashbrooke, Sunderland, SR2 7HJ, United Kingdom
}

\begin{abstract}
With a large number of railway development projects in Europe and worldwide, which once completed will be serving rail passengers of the future, this paper aims to take a step back and evaluate current railway systems performance. The objectives are to compare statistical data on various passenger-related parameters of the railway system in a number of selected European countries and draw conclusions on the level of their performance when compared to the European average.

Analyses of publically available statistical data, extracted from the Eurostat service at a European level will allow for a comparison of various indicators which influence the performance of the railway systems from an infrastructure and operational perspectives. The analyses will also allow identifying key performance indicators for the accurate assessment of the rail systems.

The paper will highlight case studies for various parameters which are important to stakeholders of the railways, including infrastructure managers, rail operators, policy makers and the end users. This knowledge will be to the benefit of today's railway industry as well as the rail systems of the future, as it will show trends drew upon existing data which might continue in the future.
\end{abstract}

Keywords: rail and logistics, evaulation, statistical analysis, European rail systems

\section{Introduction}

Over the last few years the railway system in many European countries has come under increasing criticism within the media with many people complaining about capacity issues, delays, cost etc. The general complaints are that most trains are late and overcrowded, especially during the rush hours when people are commuting (United Kingdom-based headlines: BBC, 2014; Telegraph, 2013; Rail Technology Magazine, 2015; The Guardian, 2014) thus feel disappointed by the unreliable system. This forms the basis of this paper, which will look at whether the rail system within Europe is really as bad as the media sometimes portrays by benchmarking rail system performance of few cautiously selected European countries.

\section{Methodology}

\subsection{Data source}

To evaluate the passenger rail system performance at a European level a reliable data source is needed. Eurostat (2016), the statistical office of the European Union (EU), holds a range of data for many different parameters (e.g. population, industry, transport) that enable comparisons between European countries and regions. The main reason for using Eurostat data in the paper is that it is a very convenient system, storing a large number of, relevant in this case, rail-related data available in a single location for most of the countries within Europe. Moreover, the system is supplied with data collected and harmonized by The National Statistical Institutes of the Member States of European Union (Commission of the European Communities, 2009) meaning that the source is reliable. And above all the Eurostat database is free to use and offers open access to its data which makes Eurostat superior to some other data sources, which are either available at national level only (e.g. data.gov.uk service in the United Kingdom) or require an access charge (e.g. UIC statistics). Therefore, Eurostat is clearly a suitable data source and will be the main resource of data used for analyses within the paper. 


\subsection{Plan for data analyses}

The data imported from Eurostat (2016) will be used to produce graphs showing trends within the data in order to make comments on the state of the current European passenger rail systems. First, for majority of the parameters, the average value (arithmetic mean) of the European countries for a particular year will be plot on a graph. Next a graph with values for a selection of European countries will be produced to see if the average trend for Europe is similar or different to the trends for the individual countries. Majority of data gathered will cover years from 2004 to 2014, as available in Eurostat, although some data is available for other periods. The set of $10+$ year data period is large enough to allow for a reliable look for any trends in the data gathered.

\subsection{Selection of sample countries}

The country sample is selected according to three-folded criteria: a level of Gross Domestic Product representing economic situation in a country, a position in the Rail Liberalisation Index 2011 being a reflection of a country's progress with rail liberalisation issues and a location at the 2015 European Railway European Performance Index demonstrating a combination of intensity of use, quality of service and safety issues in a country's railway sector. A selection of countries with high, medium and low values of the above criteria, reflecting the state of the railways across Europe, will be considered.

\subsubsection{Gross Domestic Product}

Values of Gross Domestic Product (GDP) vary within the European countries. The GDP is defined as "the total value of the final consumption expenditures of households, non-profit institutions serving households and general government plus gross capital formation plus the balance of exports and imports" (Eurostat, 2016b). Also, the volume index of GDP per capita in Purchasing Power Standards (PPS) is, in this case, expressed in relation to average for the European Union (28 EU member states) which is set to equal 100. Therefore if the index of a country is lower than 100 , the level of GDP per head in this country is lower than the average for the EU and consequently if the index of a country is higher than 100 then this country's GDP per head is higher than the EU average.

Figure 1 displays values for GDP per capita in PPS for the period from 2003 to 2015 with average values for the 13years period for each country displayed above bars on the graph and later in the text in brackets. The GDP results are divided into five groups with the bottom group representing values up to 25 and then each next group with up to 25 scores more with the top group reaching values between 152 and 255. It can be seen that the exceptionally high GDPs above 150, located on the left hand-side of the graph, are presented for Luxemburg (255), Norway (175) and Switzerland (152). Twelve other countries, including small-size countries like Belgium (118) or Netherlands (134), medium size countries like United Kingdom (114) and large-size countries like Sweden (125) and Germany (120), all have the indexes above the EU average ranging from a steady just above 100 points for France (108) to nearly 150 points for Ireland (137) until 2008. Italy (103) and Spain (97) results position the two countries at the border of the EU average of 100. Majority of countries classified in the bottom two GDP groups, with GDP values up to 69, joined EU relatively recently (year 2004 or later). Interestingly, GDP indexes for Balkan-based countries are mainly below or around 50 with medium-sized Bulgaria (42) and Romania (46) showing a rapid growth of 10 and 20 points, respectively, from the level of $30+$ in 2003.

In order to see whether wealthier countries and poorer countries fit in with the general trend across Europe or whether there is some disparity for wealthier or poorer counties in terms of their railway systems performance, a selection of countries with a range of GDPs will be chosen as the final sample. 


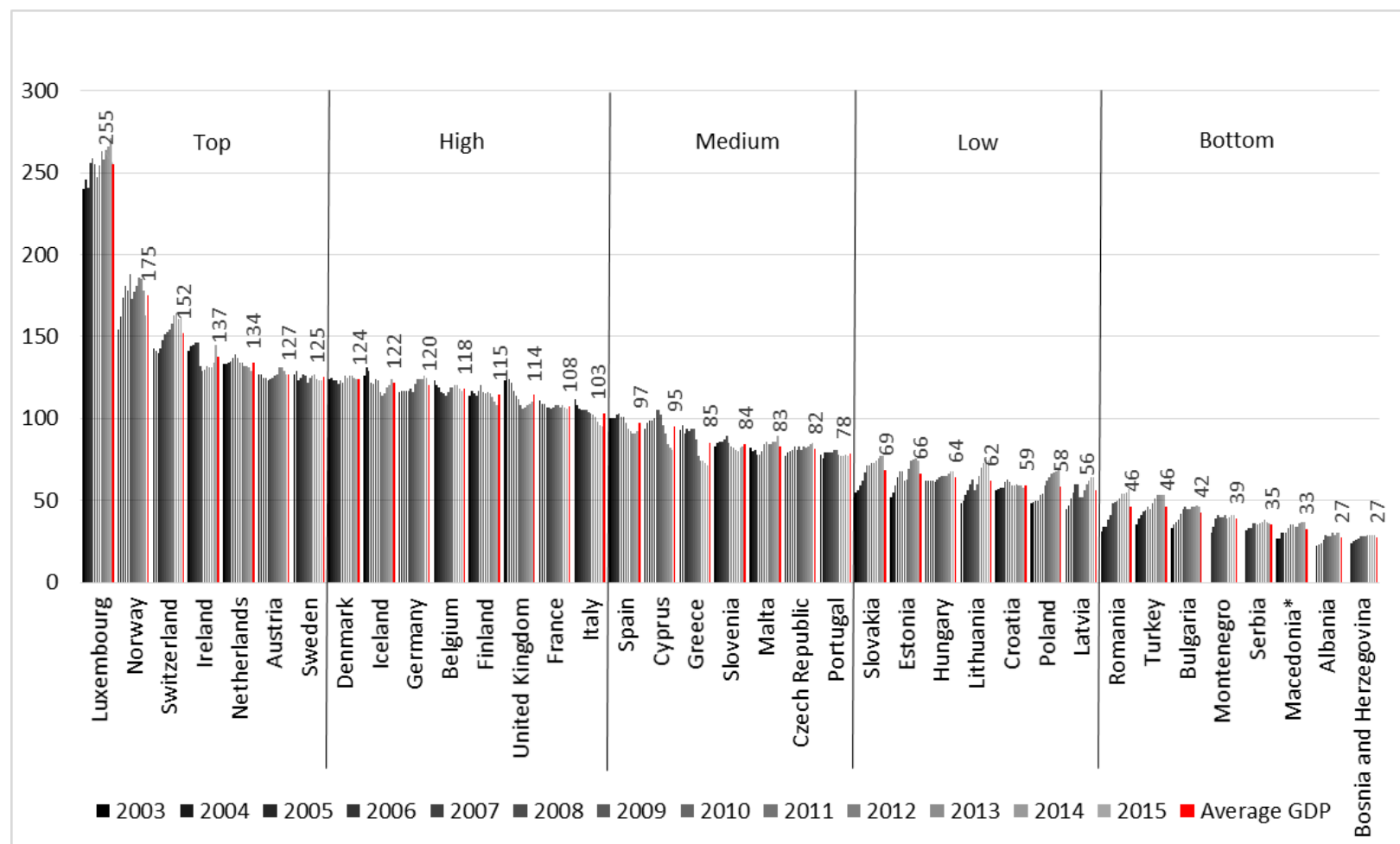

Figure 1. GDP per capita in PPS for European countries [count]. Data source: Eurostat (2016)

* the Former Yugoslav Republic of Macedonia

\subsubsection{The Rail Liberalisation Index 2011}

Results for the European countries published in Rail Liberalisation Index (LIB Index) supply information on the progress with liberalisation process in the European railways and take into account passenger and freight rail transport (Kirchner, 2011). Primary data for LIB Index is extracted from various sources, including but not limited to national statistical offices, Eurostat, IBM Global Business Services Network, current studies and annual reports, which ensure the reliability of the data. The LIB Index is composed of two other indexes where $80 \%$ of weight is put on ACCESS Index and the remaining 20\% on LEX Index. LEX Index, characterised as 'law-in-the-books', describes market entry legal requirements and the level of support external Railway Undertakings receive from regulatory authorities and is composed of three sub-indices: organisational structures of the incumbent $(25 \%)$, regulation of market access $(45 \%)$ and competencies of the regulatory body $(30 \%)($ Kirchner, 2011). ACCESS Index, described as 'law-in-action', analyses actual accessibility of markets and allocation procedures used and is composed of five sub-indices: information barriers (5\%), administrative barriers (20\%), operational barriers (45\%), share of accessible domestic market (25\%), sales services in passenger transport (5\%)(Kirchner, 2011).

The final LIB Index 2011 results for the European countries are displayed on Figure 2. It can be seen that countries are grouped into three categories: advanced ( 6 countries), on schedule (15 countries), and delayed (6 countries). The six countries classified as Advanced are all EU members, represent Western and Northern Europe and have a GDP of over 100, as presented on Figure 1. However, the 'delayed' group of countries represent a mix of high, medium and low GDPs. 


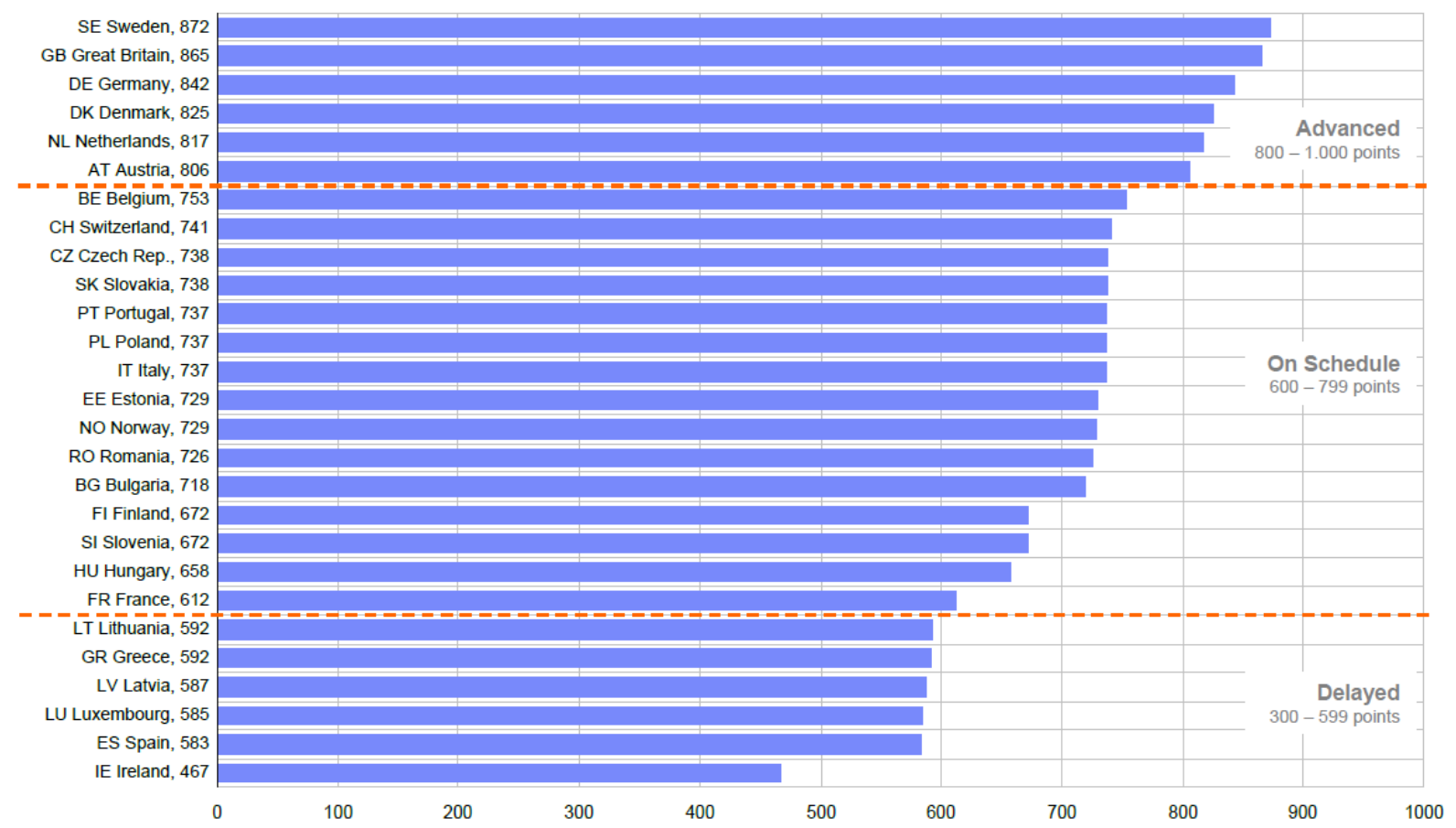

Figure 2. LIB Index 2011. Source: Kirchner (2011)

\subsubsection{The 2015 European Railway Performance Index}

The 2015 European Railway Performance Index (RPI) aims to compare performance of the European railways, for both passenger and freight traffic, based on a multi-criteria with the primary data sourced from the International Union of Railways (UIC) 2012 database (Duranton et al., 2015). The RPI is composed of three equally weighted elements: intensity of use, quality of service and safety of a railway system studied. Each of the three components includes between two and four parameters which form its final value. For example, the intensity of use criteria includes passenger volume (50\% weight) and goods volume (50\% weight), quality of service element consists of punctuality of regional (25\%) and long-distance trains (25\%), percentage of high speed rail (25\%) and average fare (25\%), all in suitable and unified units. In the context of this paper the RPI is taken into account as it clearly measures and displays performance of each of the European rail system and allows for a comparison between individual countries. Figure 3 shows results for the 2015 RPI with countries divided into three groups of: Tier 1 with the highest, Tier 2 with medium and Tier 3 with poor performance results. It can be seen that all but one countries classified in Tier 1 and Tier 2 are representing Western and Northern Europe with the best performance scores given to non-EU member Switzerland (7.1 score) and EU member state Sweden (6.6 score). Results for Tier 2 countries are showing scores between 5.8 for Austria and 4.6 for Norway. Slovenia with a score of 4.3 opens a group of Tier 3 countries. However, what is striking are very poor scores for Bulgaria for all three components of the performance index, which once summarised to a score of 2.2 put this country at the end of the European rail performance spectrum. 


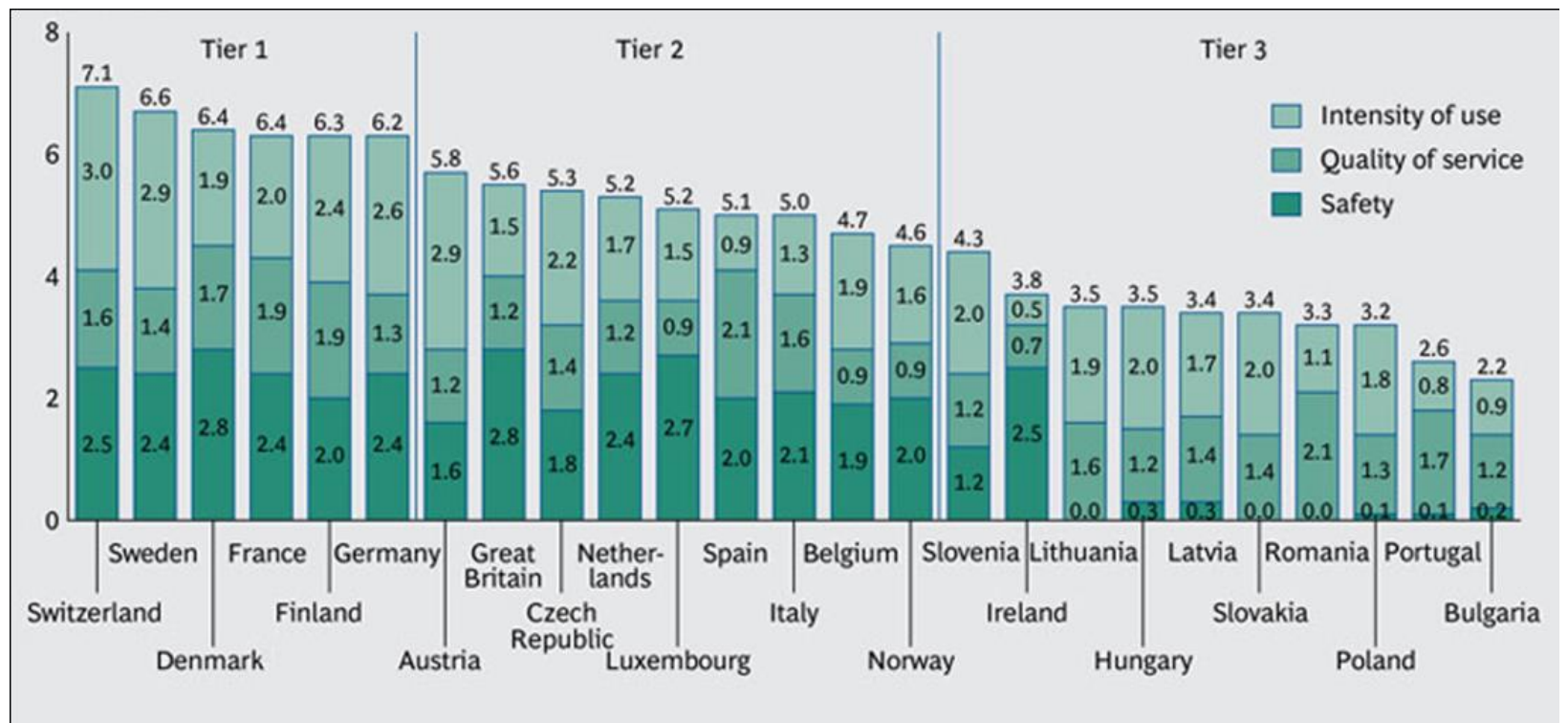

Figure 3. The 2015 RPI. Source: Duranton et al. (2015)

\subsubsection{The sample countries}

Based on the multivariate criteria presented earlier the selected sample includes a mix of five EU countries with one top, three middle and one low positions in the rail indexes. The reason for this selection is to analyse data for a spectrum of European countries, from well advanced to still developing economies. The selected sample countries are:

- Sweden (high GDP, Advanced LIB 2011 Index, Tier 1 in the 2015 RPI),

- United Kingdom (medium GDP, Advanced LIB 2011 Index, Tier 2 in the 2015 RPI),

- Italy (medium GDP, On Schedule LIB 2011 Index, Tier 2 in the 2015 RPI),

- Slovenia (medium GDP, On Schedule LIB 2011 Index, Tier 3 in the 2015 RPI),

- Bulgaria (low GDP, On Schedule LIB 2011 Index, Tier 3 in the 2015 RPI).

Since the five countries are selected for further investigation their individual GDP is examined so that at a later stage this could be linked to rail-related performance indicators. Therefore Figure 4 presents GDPs of the five selected countries with the results as follows: Sweden (above 120 and steady), United Kingdom (around 110, but decreasing), Italy (just above 100, but decreasing), Slovenia (above 80 and quite steady), and the poorest out of the five results for Bulgaria (around 40, but increasing). This shows that although all five selected countries are members of EU, their GDP can vary significantly, which is also expected to influence their level of rail system performance. 


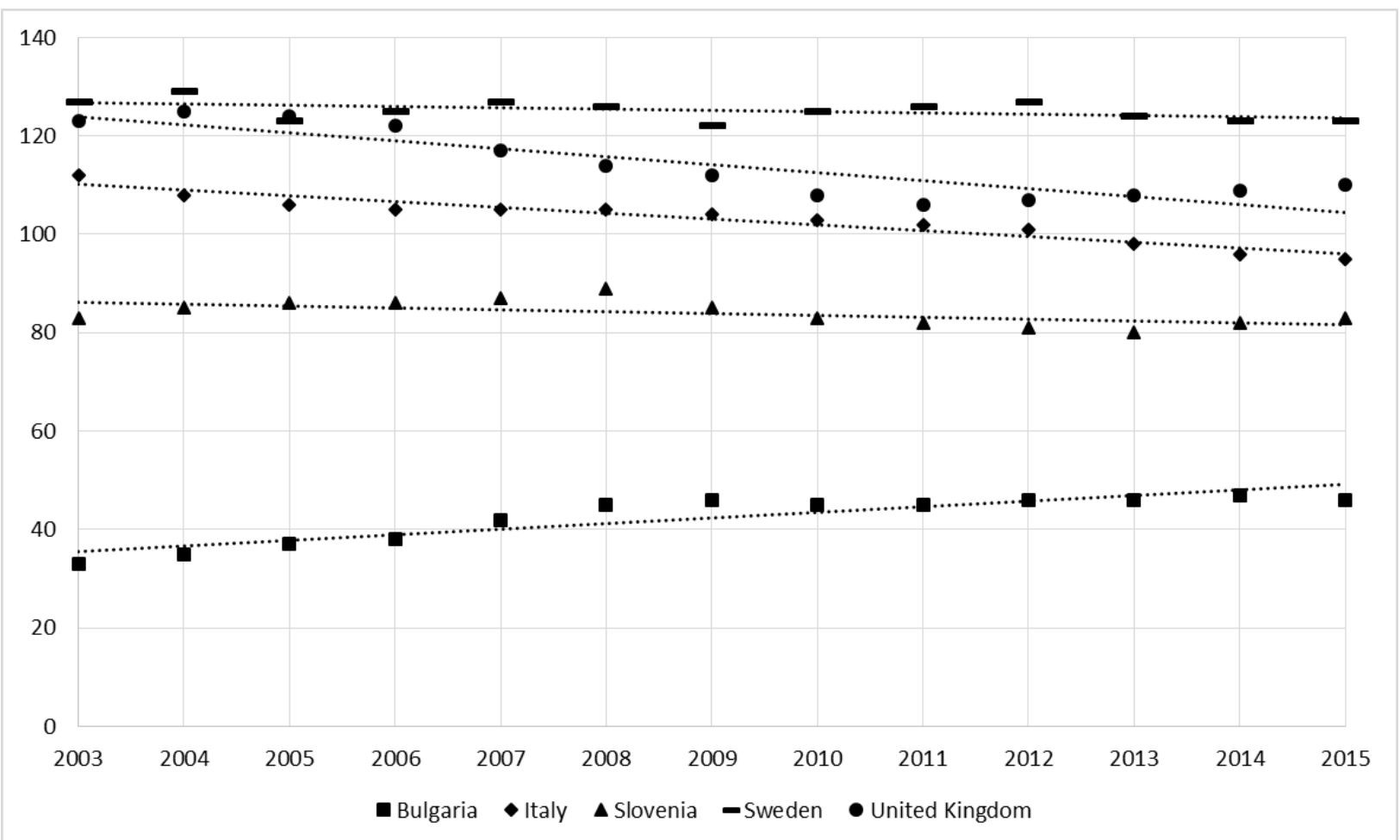

Figure 4. Gross Domestic Product per capita in Purchasing Power Standards for the sample countries. Data source: Eurostat (2016)

In addition to GDP, investigating two more parameters such as a country size and a country population will shed more light on understanding railway data presented in later sections. Therefore Figure 5 displays values for the five selected countries, in terms of their size [1,000 km2] and population [million], on one graph. Slovenia, the smallest country out of the five selected, has the smallest population of approx. 2 million people. However, the greatest difference in area $\left[1,000 \mathrm{~km}^{2}\right]$ vs. population [million] ratio is recorded between Sweden (approx. 45:1) and the United Kingdom (approx. 4:1) meaning that population is much more dense in the latter country (65 million people live on 243,000 $\mathrm{km}^{2}$ ). It is expected that this fact will be reflected in KPIs presented in the analysis part, e.g. area vs. population ratio will impact on volume of passengers as well as on investments in rail infrastructure. 


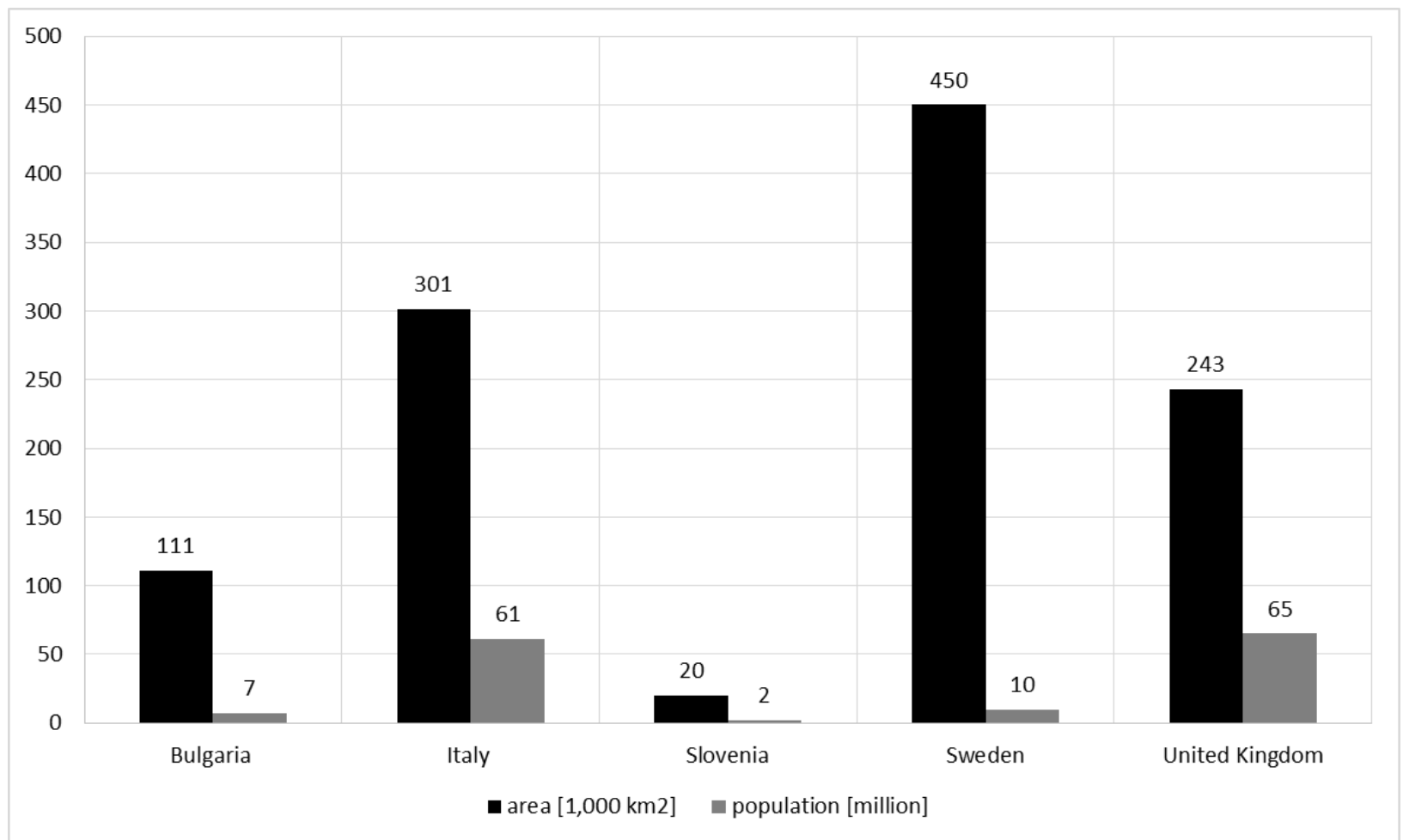

Figure 5. Country area $\left[\mathrm{km}^{2}\right]$ vs. country population [million people] for the sample countries. Data source: Eurostat (2016)

\subsection{Selection of Key Performance Indicators}

The paper will focus of rail datasets available openly via Eurostat (2016) which, as a European governments' supported service, guarantees data reliability. In terms of rail-related data Eurostat (2016) offers variety of sets on: railway equipment (number of locomotives, railcars, wagons, etc.), railway enterprises (type of enterprises, employment data, expenditure, etc.), railway traffic (train-movements, traffic flows, etc.), passengers and freight measures (passengers and goods transported), and accidents (number of victims, accidents, etc.). Although Eurostat (2016) uses data supplied by national statistical institutes, there are still some missing values for some periods in the datasets. This issue will be monitored closely when selecting KPIs and displaying data on graphs.

As the paper is focused mainly on passenger rail, the final dataset selected for analysis consist of four main Key Performance Indicators (KPIs): length of rail track, annual number of rail passengers, annual number of rail victims and numbers of rail seats and berths. It is expected that the analysis of the four KPIs and their trends will give a reasonable overview in making an evaluation of the European passenger rail system.

\subsubsection{KPI 1: Length of rail track}

The first parameter to be discussed within the paper will be length of track. This will be a suitable topic to use as an indicator on the performance of the rail system within Europe as it will show whether the countries within Europe are increasing the accessibility of the rail system, allowing more people to access the rail system from potentially more isolated locations away from cities/popular lines. Thus, any trends in the graphs for the length of track will be a suitable indicator on whether the European countries are improving their rail systems, improving its performance. The length of track will be also compared with length of motorways to see whether those match the trends in the railway sector.

\subsubsection{KPI 2: Annual number of rail passengers}

The second topic to be discussed is the annual number of passengers. This will be a suitable indicator of whether European rail systems are improving as an increasing number of passengers could be used as an indicator on the 
popularity of the rail systems within Europe or that an increasing number of people are relying on the rail system in order to get to work etc. This parameter will be compared with the capacity of rail vehicles to see if the rail operators are increasing or decreasing the capacity of railway vehicles to deal with an increase or decrease in passenger numbers. In addition, this parameter will be compared with a modal split for rail passengers to see whether trends in modal split reflect trends in the railway sector itself.

\subsubsection{KPI 3: Annual number of victims}

The third topic to be discussed within the paper is the annual number of victims. Average annual number of rail victims in Europe will be compared against time and then in more detail the statistics for rail victims will be divided into rail injuries and rail fatalities presented per country. The numbers of victims will have to be interpreted along with the volume of passengers within the country in mind as it is assumed that a small system will be causing a relatively smaller number of railway victims than a larger system with a heavier use. Thus it will be useful to see if the rail infrastructure managers are increasing the safety of the rail systems which will therefore be a good indicator on whether the rail systems within Europe are improving in performance.

\subsubsection{KPI 4: Seats and berths}

The final topic to be discussed within the paper will be the capacity of railways in terms of seats and berths. This will be a good indictor on whether the European rail systems are improving in performance as an increasing number of people are complaining about how full trains are and how this makes any journey at peak times very uncomfortable (The Guardian, 2014). It will be interesting to see whether the rail operators are listening to this feedback and addressing passenger concerns in order to improve the performance of the rail system by increasing seats capacity and as a consequence improving consumer satisfaction.

\section{Analysis of Data}

Majority of data presented in this section is sourced from Eurostat (2016), although in few specific cases some international UIC (2014) data was employed to set the context for Europe-related analysis. To minimize distortions in the data presented, which might occur due to variability of countries' dimensions, additional parameters, such as country size and country population, will be taken into account when analysing trends of the selected KPIs'.

\subsection{Length of track}

Before analysing trends for length of track in Europe and in the five selected countries it will be useful to compare the values of this KPI at an international scale. International Union of Railways (UIC) publishes various data (mainly available for a fee) related to rail statistics worldwide, including data on the length of rail track. Selected open data available from UIC (2014) was plotted on a graph and Figure 6 presents the total length of rail track for five geographical regions, including Europe. It can be seen that the highest results of nearly $400,000 \mathrm{~km}$ are recorder for America, and they are achieved most likely due to the continent size and popular rail freight services in operation. Africa however, which shows the lowest results, most likely to its difficult economic situation, recorded an impressive increase in the length of track from approx. 52,000 km in 2006 to approx. 70,500 km in 2013. Europe's results place the old continent in the center of the graph, even higher than Asia, although the data for both continents show very similar steadily increasing trends. However, rail investments in Asia are progressing quickly and it is expected that by 2020 China itself will build 30,000 km of a new (including high speed) rail track (CCTV, 2016) contributing to an increasing trend for the length of track in Asia itself. 


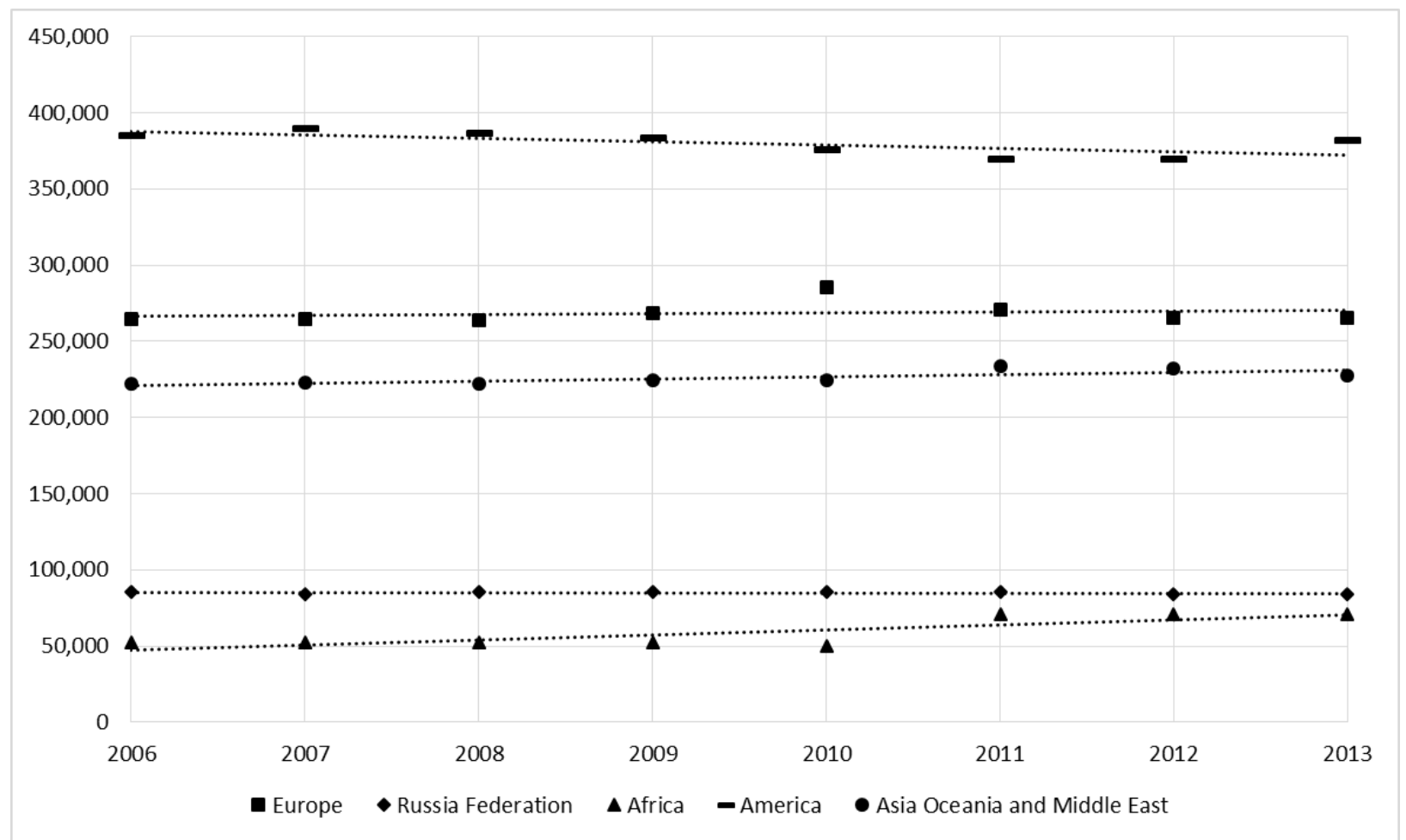

Figure 6. Total length of rail track (kilometers) over time [km]. Data source: UIC (2014)

To investigate situation in Europe further more detailed data on the length of rail track recorded from 2004 to 2014 for various European countries was collected from Eurostat (2016). The length of track was measured and recorded in kilometers and Figure 7 shows the plot of the average length of track of the European countries.

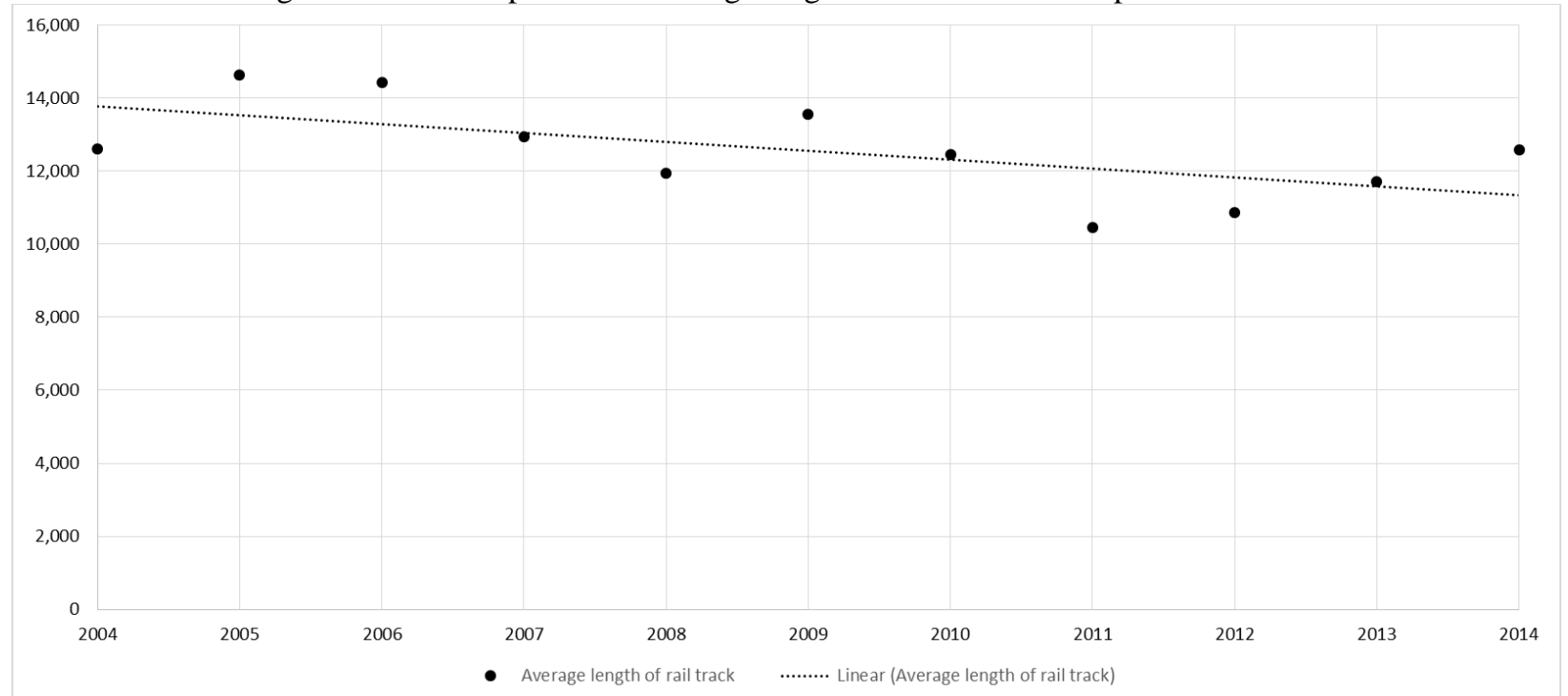

Figure 7. Average European length of rail track against time per country [km]. Data source: Eurostat (2016)

It can be clearly seen on Figure 7 that there is a negative trend for the average length of rail track in Europe, although the values form a shape of a sinus wave on the graph. This phenomena can be explained in two ways: firstly, 
construction of new lines in some years as well as closures of lines in the others cause fluctuations; secondly there are gaps in Eurostat (2016) data (see gaps on Figure 8) which contribute to the visible fluctuations in the displayed results. Nevertheless the data suggests that Europe lost about 2,000 km of track within the last decade, mainly due to structural changes in the railway sector and line closures.

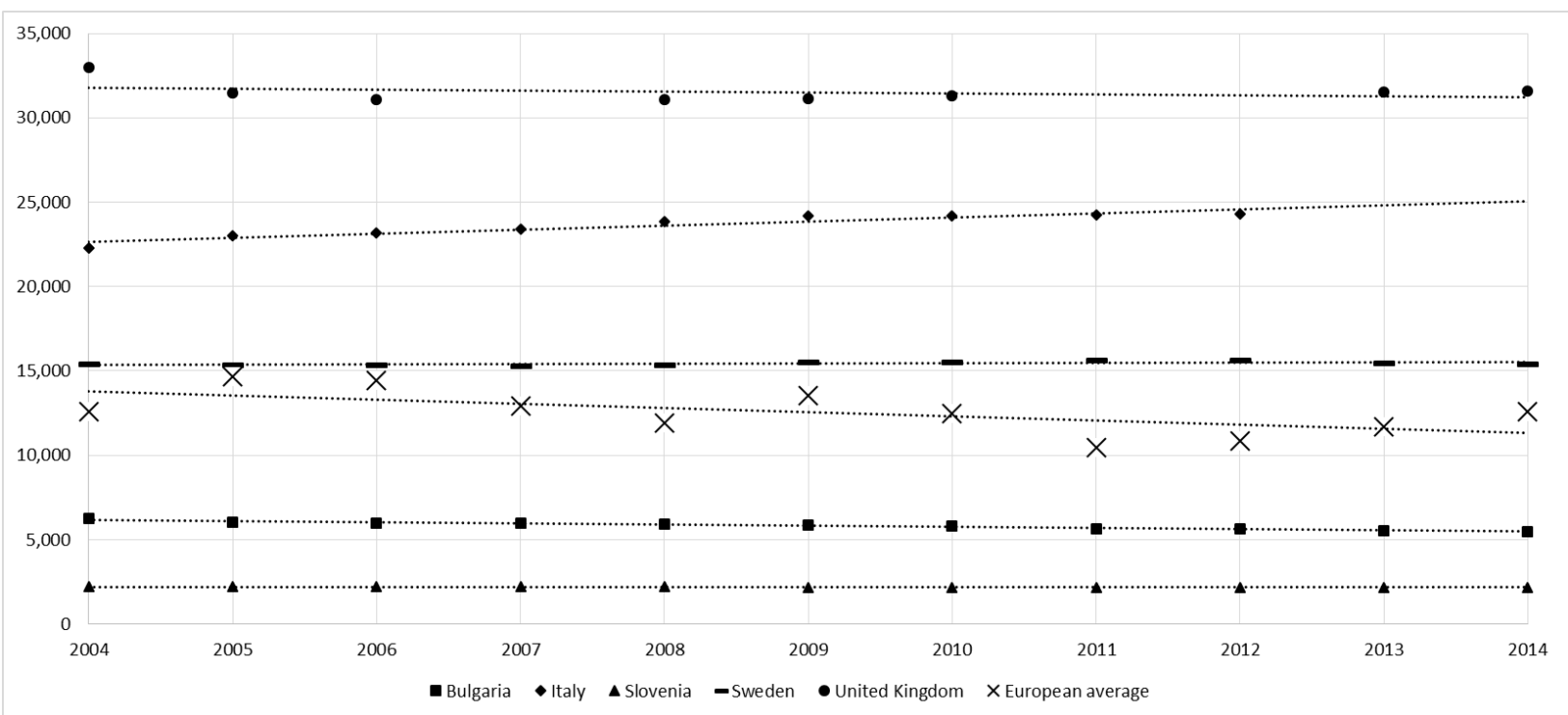

Figure 8. Length of rail track against time for the sample countries [km]. Data source: Eurostat (2016)

As the five countries have been selected for further investigation, Figure 8 shows detailed results for the sample and the European average for a closer comparison. It is clearly seen that results for the United Kingdom are the greatest (over 30,000 km) out of the five and results for Slovenia are about ten times smaller (around 3,000 km) than the United Kingdom's values. This is obviously due to those countries sizes in terms of area and population. However, Figure 7 shows very similar trends within the sample countries for the length of track parameter. All three countries: the United Kingdom, Slovenia and Bulgaria have negative trends, as time increases the length of track in these countries decreases (there is a negative correlation for these three countries). Interestingly, Italy and Sweden have an increasing length of track, although the increase is much more dynamic in Italy than in Sweden $(22,277$ vs. 15,381 in 2004 to 24,277 vs. 15,601 in 2012, respectively).

Anyhow, the detailed Figure 8 gives a rather different view to the average shown on Figure 7 as it indicates that not every country in Europe has a decreasing length of track. Furthermore, the increasing length of track might be a sign of countries attempting to grow their economies by extending the length of track and improving connectivity within their borders. This can occur as extending the length of track, followed by more rail services on offer stimulates economy as people are employed to build the railways, maintain it as well as more customers can use it. Indeed Figure 1 showed that the economies of both Sweden and Slovenia are quite stable and the Bulgarian economy has been increasing over similar time period whereas the United Kingdom's economy is decreasing showing there may be a causal link.

Although, the extent of the relationship between GDP and length of track is beyond the scope of this paper it is worth stressing that there has recently been a major economic recession in 2010 for the British economy, this probably affected the results presented on Figure 8. It must be highlighted here that many factors lead to economic growth of a country and correlation does not always imply causation. Therefore, the extent of the relationship between the changes in length of track and GDP should be treated with a sufficient care.

In order to put the length of track in the context of other transport modes and investments Figure 9 shows the length 
of motorways, which is compared with Figure 7. However, due to missing values data for years from 2004 to 2012 is displayed only. It can be seen from Figure 9 that there is a strong positive correlation between the two variables; as time increases, the length of motorways generally increases. This is in contrast to the length of track presented on Figure 7 which has been decreasing over time. This shows that governments who are usually a body responsible for funding infrastructure projects within countries generally are investing in building new motorways rather than in building new rail tracks or maintaining the existing infrastructure. One explanation for this state could be that governments usually prefer to invest in a financially cheaper option and motorways have a priority over rail infrastructure developments then.

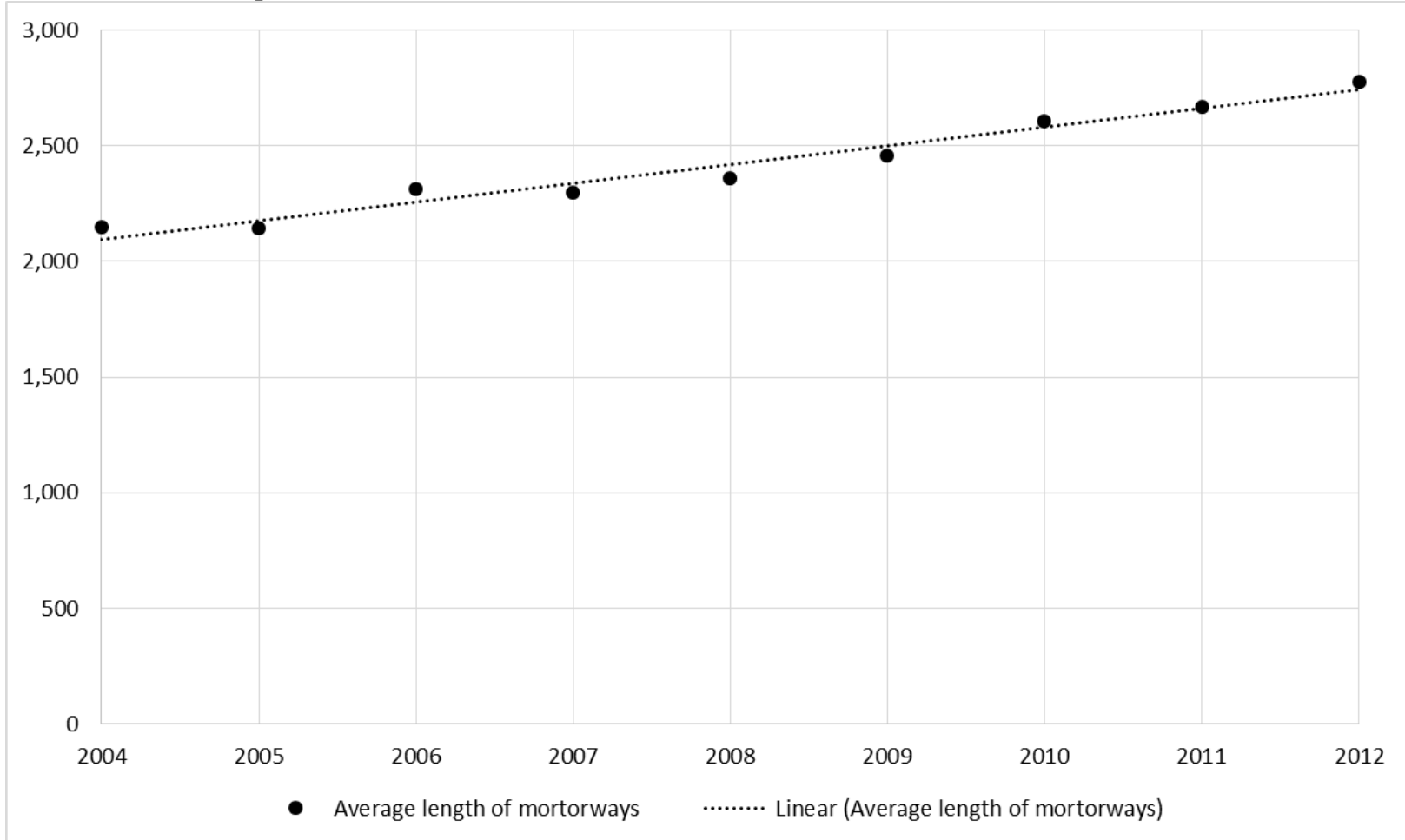

Figure 9. Average European length of motorways per country against time [km]. Data source: Eurostat (2016)

Although the European average length of motorways per EU member is about 2,500 km, Figure 9 displays results for the sample with Italy scoring the highest with nearly 7,000 km, United Kingdom scoring medium with just under $4,000 \mathrm{~km}$ and Bulgaria scoring lowest with about $500 \mathrm{~km}$ only. These results reflect the countries size, with Slovenia and Bulgaria being the smallest size countries and their economies also being at the lowest level (see Figure 1) out of the five. Overall, trends for the five countries are slightly positive with an exception of the United Kingdom where results have been rather constant between $2004(3,657 \mathrm{~km})$ and 2011 (3,685.7 km) (no value for 2012 available).

The results for the length of rail track and the length of motorways in the five countries sampled show that in general there is a slightly positive trend for investments in the motorways whereas railway infrastructure investments show a rather negative trend over the period studied. According to the figures only Italy has demonstrated a positive trend in the length of rail track within the last decade. Although not shown by the data the United Kingdom is planning a large investments into high speed rail (HS2) and aims to have an extra of $225 \mathrm{~km}$ of track by 2026 (Department for Transport, 2012). Although no information about plans for similar rail investments in the other four sample countries is publicly available, in 2014 a Joined Undertaking named 'Shift2Rail' was launched in Europe. Shift2Rail, as a partnership between industry and European Commission, is offering research funding for ideas encouraging and simplifying a shift from road to rail for both passenger and freight transport (Shift2Rail, 2016), and all the five sampled countries as EU members can get involved in it. 


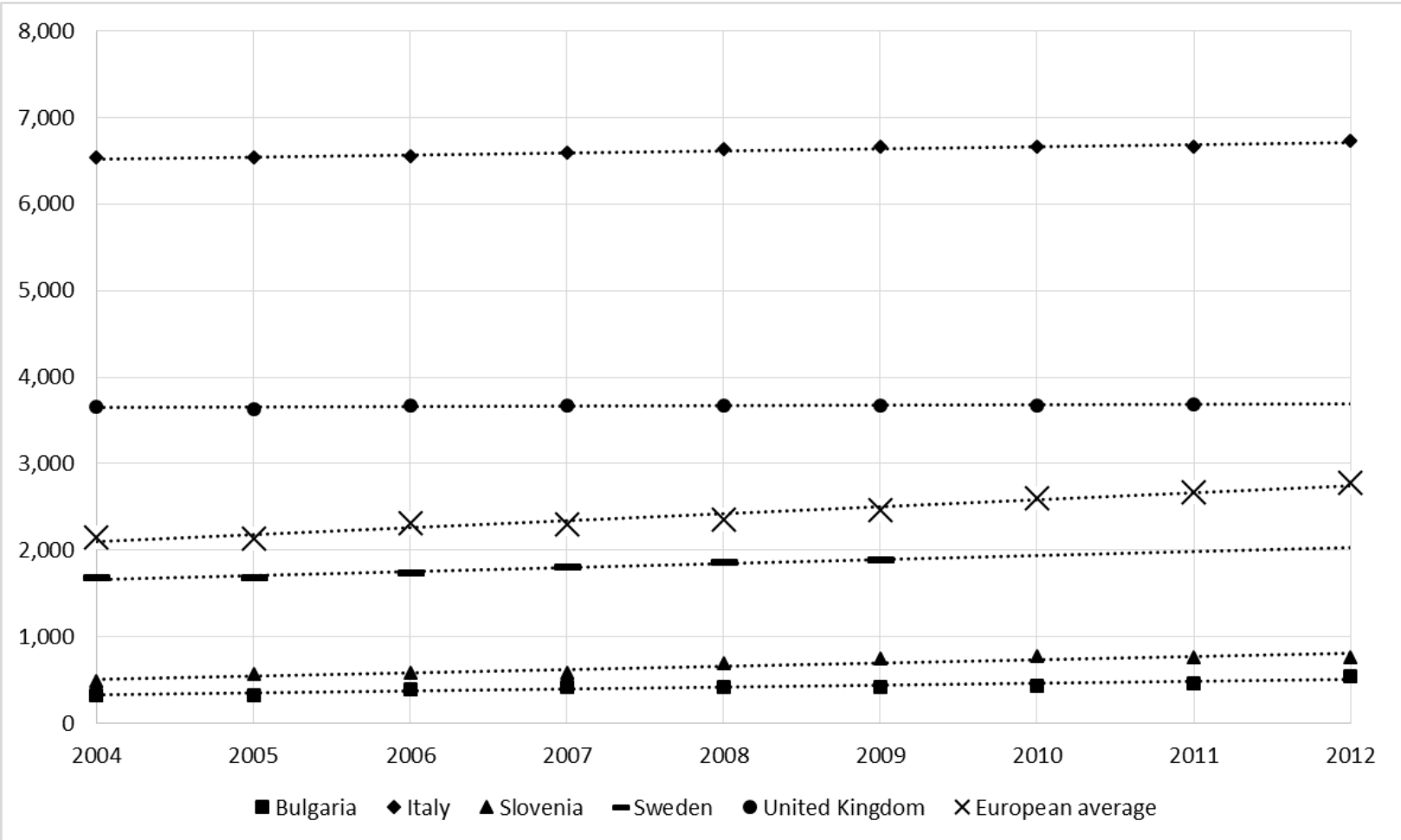

Figure 10. Length of motorways (sample) against time for the sample countries [km]. Data source: Eurostat (2016)

\subsection{Annual Passenger numbers}

The results for rail passenger-kilometres for five different geographical regions worldwide are provided by UIC (2014) and displayed on Figure 11. Rail passenger-kilometre is a "unit of measurement representing the transport of one rail passenger by rail over a distance of one kilometer" (Eurostat, 2016b). It is striking that the results for Asia are far higher than the results for the other four regions and the trend is positive. This could be explained by differences in population numbers, which are very high in Asia (e.g. populations of China and India are the highest worldwide) in comparison to the other regions. Anyhow, results for Europe are about four times smaller than for Asia and quite steady with just under 500 billion passenger-kilometres every year. 


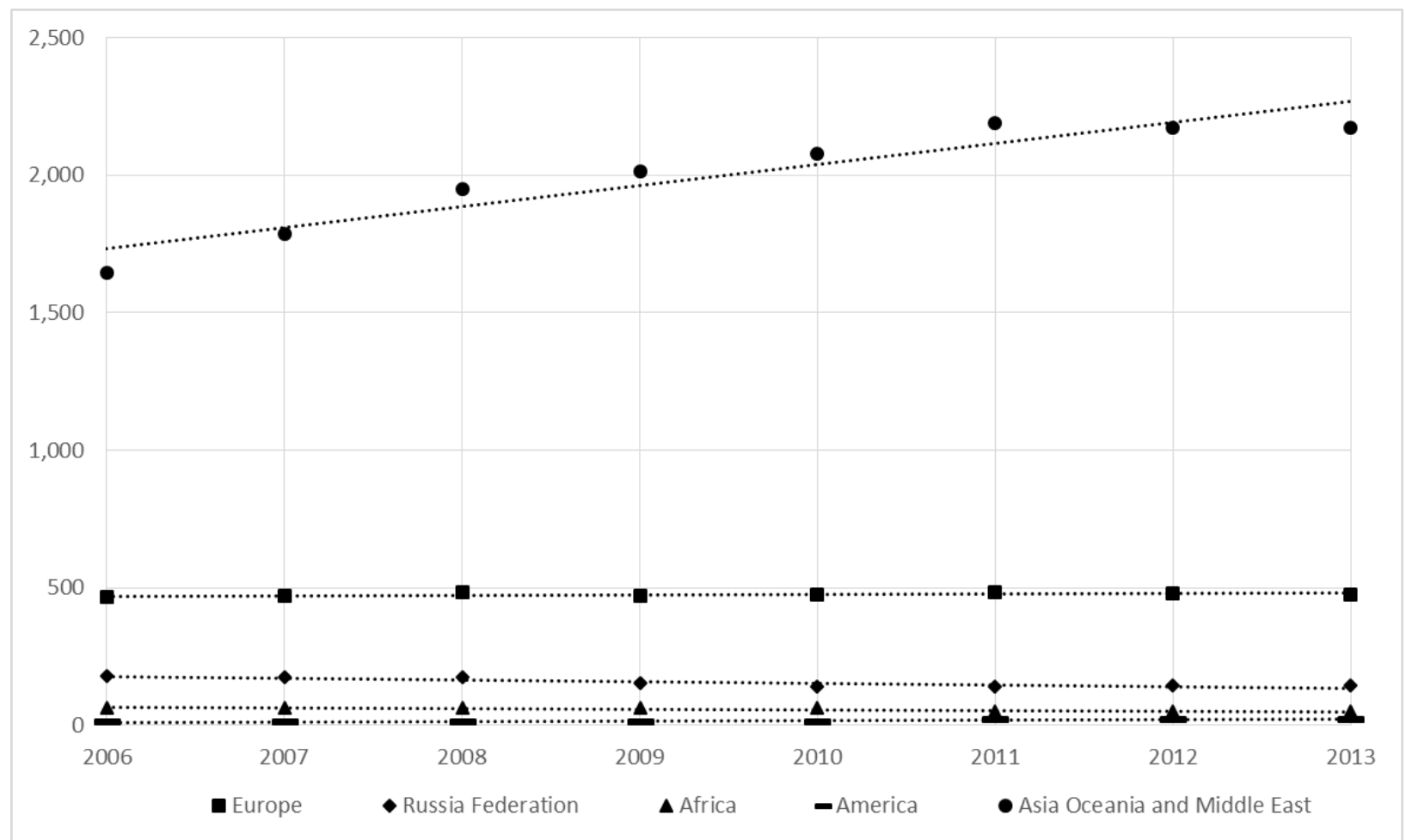

Figure 11. Rail passenger-kilometres (billions) over time for the sample countries [count]. Data source: UIC (2014)

Next, data for the annual passenger numbers, from 2005 to 2014 for various European countries, was collected from Eurostat (2016). The annual number of passengers was measured in 1000's of passengers. Figure 12 shows the plot of average annual number of passengers per European country, after removing outliers for 2010 and 2011. 


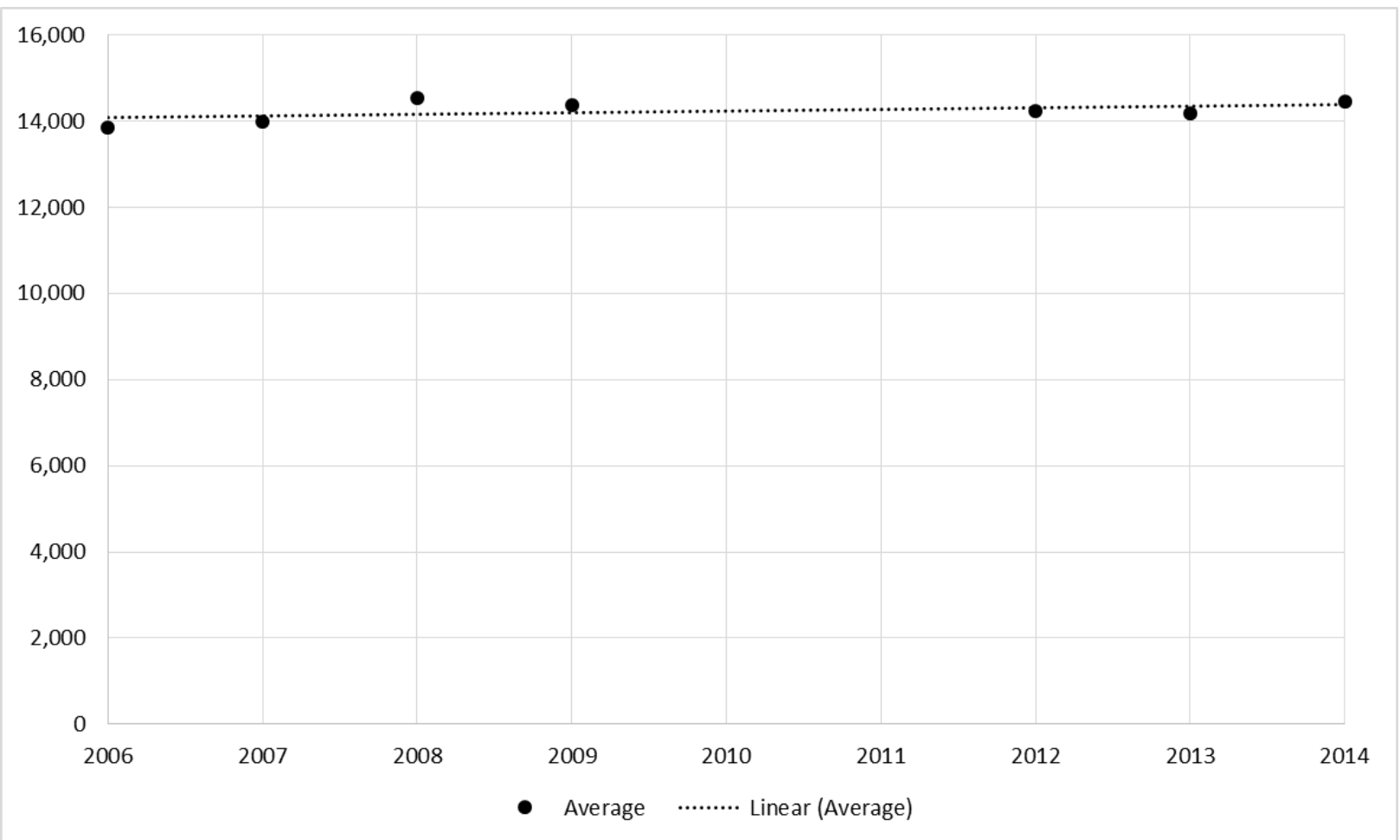

Figure 12. European country average of millions of passenger-kilometres against time [count; million passenger-km]. Data source: Eurostat (2016)

With the outliers removed the trend visible on Figure 12 is slightly positive. The new graph shows that there is a positive correlation between the annual number of passengers and the increasing of year meaning that generally the number of passengers seems to be on average slightly increasing in European countries over time. Therefore, as more people need the rail system, the rail system needs to perform efficiently and successfully to cope with the increase passenger numbers. 


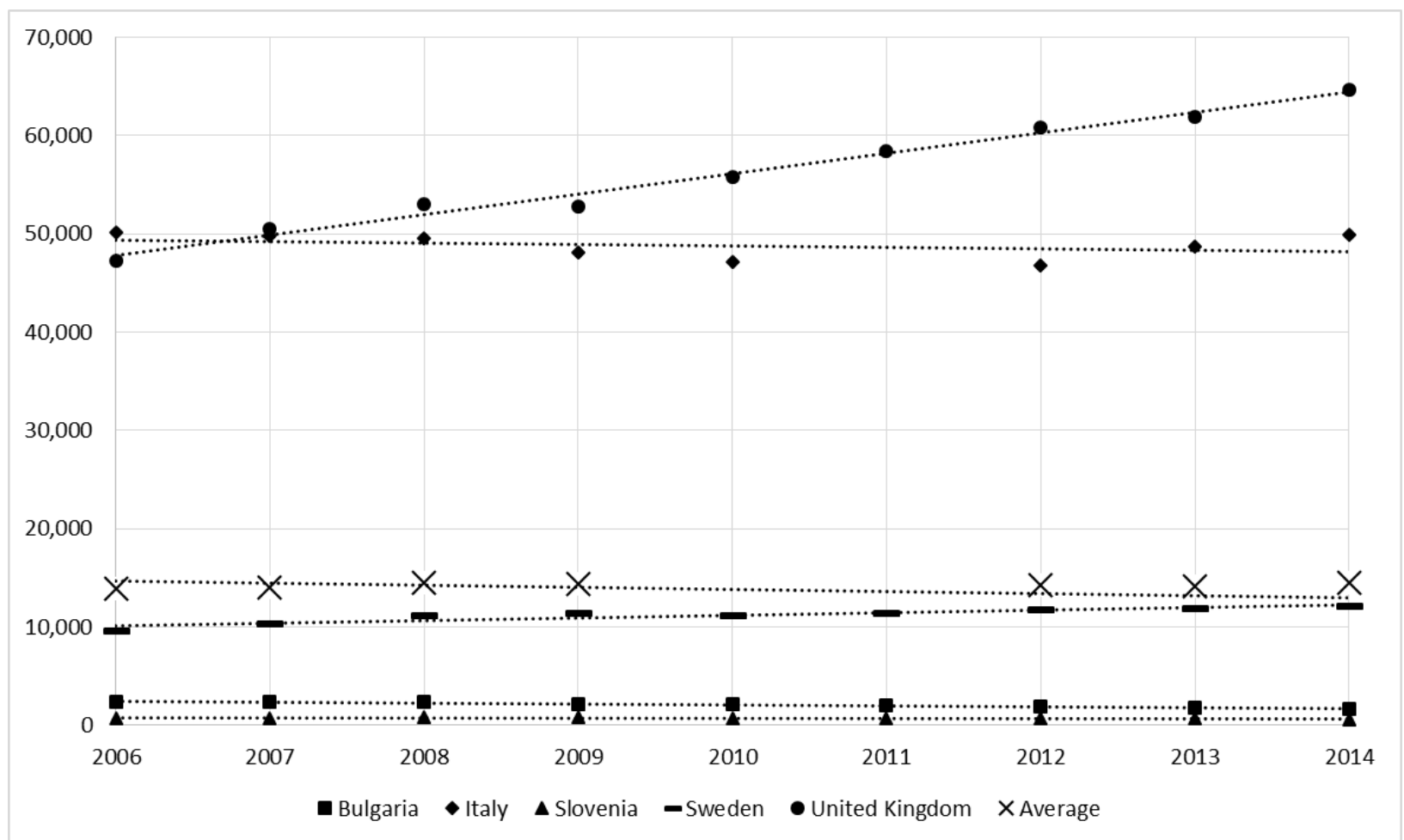

Figure 13. Millions of passenger-kilometers in sample countries against time for the sample countries [count; million passenger$\mathrm{km}$. Data source: Eurostat (2016)

In order to look at the sample's annual millions of passenger-kilometers Figure 13 was created. It shows clearly that United Kingdom has the highest values out of the five EU member states evaluated and its trend is impressively positive. It must be reminded here that, as Figure 5 showed, population of the United Kingdom is the greatest out of the five countries considered (and keeps increasing; Eurostat, 2016), and this fact potentially can have an impact on a greater number of rail passengers when comparing against the other four sample countries. Although there is a large difference in a size of population within the five selected countries, it can be seen that the two wealthier countries, the United Kingdom and Sweden, have increasing trends showing that their passenger-kilometer numbers are increasing year by year (although the increase for each of the two countries, recorded between 2006 and 2014, varies and is approx. 2,500 vs. 12,500 million passenger-km, respectively). Values for Italy, in comparison to the European country average, are very high (just under 50,000 million passenger-kilometres), but the trend is rather steady between 2006 and 2014. The trends for Slovenia and Bulgaria are rather flat with a slight decrease of values in the time period studied (104 vs. 719 million passenger-km, accordingly). This generally agrees with the average trend as there are no any large decreases in passenger numbers, the large increase in passenger numbers in the United Kingdom outweighs the rather small decrease for Slovenia and Bulgaria. The reasons for such a large increase in the numbers of rail passengers in the United Kingdom are partly because of increase in petrol prices and passengers switch from cars to trains (Parliament, 2015). Moreover, this trend is expected to continue in the United Kingdom and the numbers of rail passengers are expected to double in this country by 2035 (Parliament, 2015).

\subsection{Modal split}

As railways are part of a wider transport system for passengers a level of modal split is relevant in the context of the rail system performance and data for the target group is investigated (a similar applies to rail freight, which is not investigated in the paper). Data for the five selected countries is sourced using Eurostat (2016) data for modal split in Europe and a new graph, Figure 14 is created. Although values for modal split data in Eurostat (2016) are available from 1990, there are many missing values which no longer occur for the selected countries from 1995 onwards. Figure 
14 displays data for passenger transport collected between 1995 and 2014 with trains, cars and coaches (includes motor coaches, buses and trolley buses) listed as passengers' transport modes. The results show that all five countries have reached the level where cars are used for $80 \%$ or more of passenger transport. Also, the data for car, train and coach split is at a similar steady level for all countries but Bulgaria. Results for Bulgaria are unique in this context showing a rapid increase of about 20\% in the modal split for cars from about 60\% in 1995 to just over 80\% in 2014. Also, this country still has the highest percentage of coach's share out of the sample countries, despite about $15 \%$ decrease in this mode's share within the 20-year period investigated. In none of the five countries sampled, apart from Bulgaria between 1995-1999, the railway share of the modal split exceeded 10\% with the lowest values recorded for Slovenia (less than 5\%) and the highest, and on the rise, for the two countries with highest GDP: Sweden and the United Kingdom (nearly $10 \%$ in 2014).

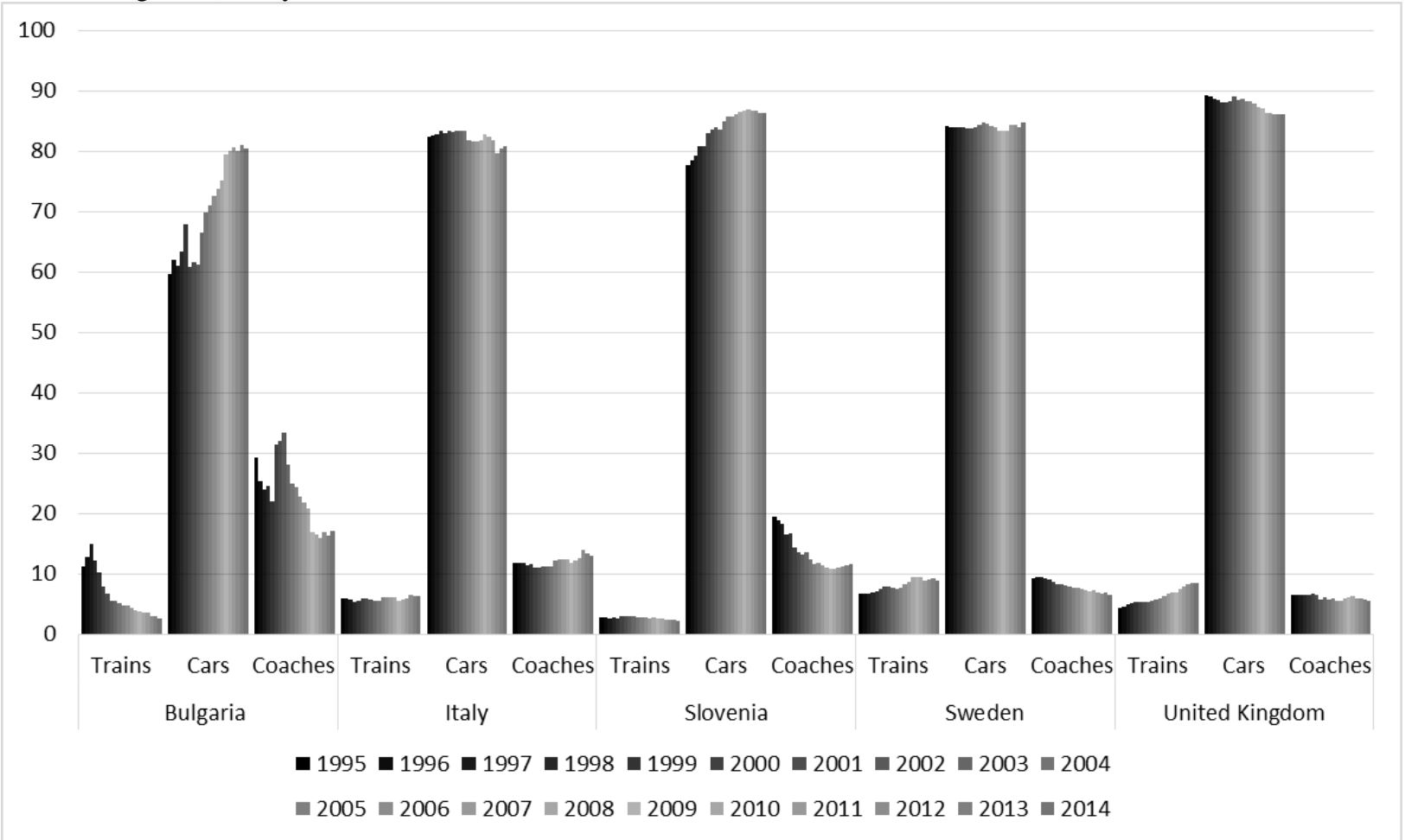

Figure 14. Modal split of passenger transport for the sample countries [\% in total inland passenger-km]. Data source: Eurostat (2016)

3.4 Annual number of victims

A set of data for the annual number of victims in railways within Europe was collected from Eurostat (2016) with the aim of comparing safety of the systems across the EU. The data collected covers the time period of $2005-2015$. Figure 15 shows the plot of the average annual number of victims for the European countries with a negative trend clearly visible. 


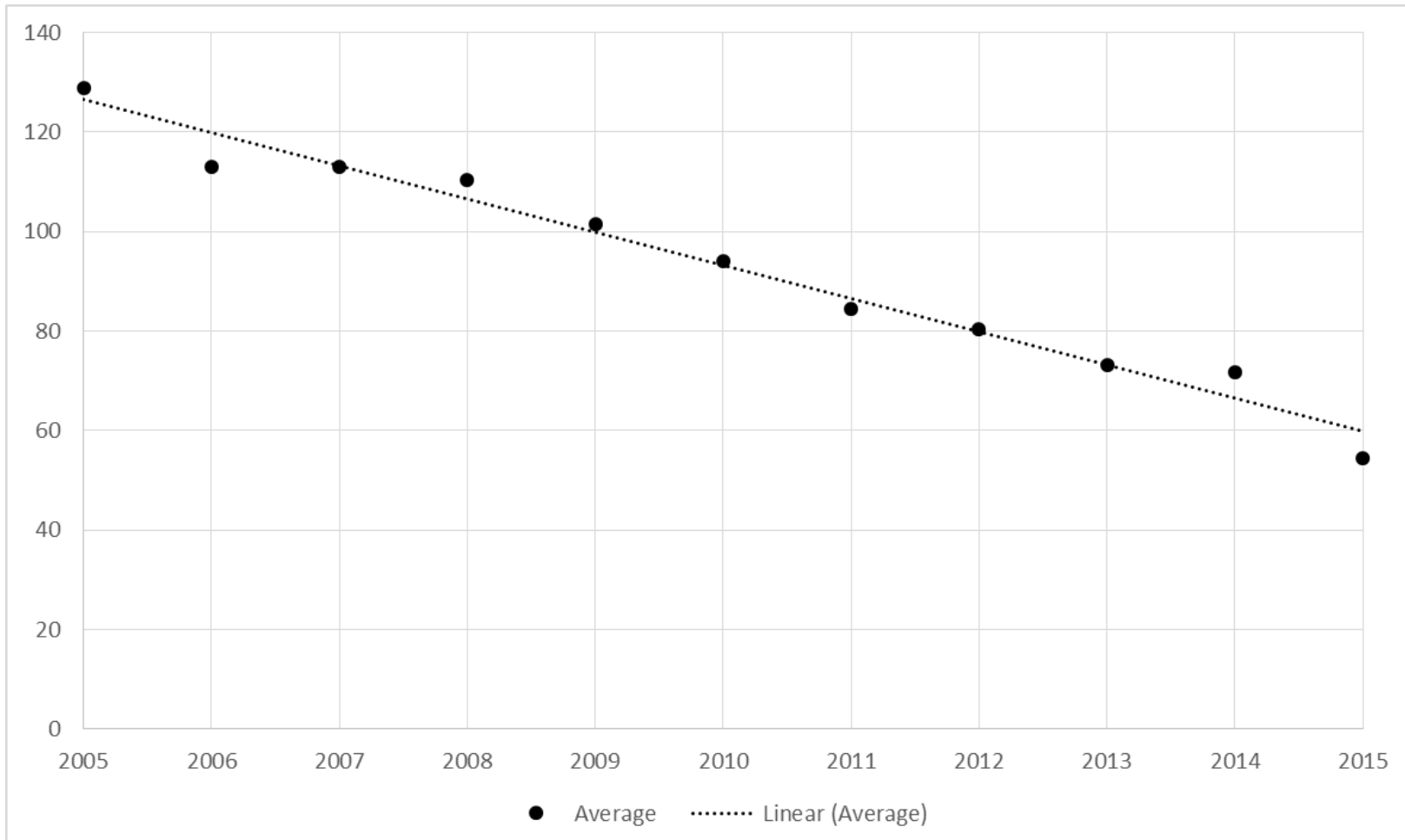

Figure 15. Average European annual number of victims against time per country [count]. Data source: Eurostat (2016)

As Figure 15 shows there is a strong negative correlation between the annual number of victims and time; as the time increases, the annual number of victims decreases. Although in some European countries this value is still very high (e.g. in Poland 494 and in Germany 334 victims a year on average) in general terms this optimistic trend shows a reduction of $50 \%$ in annual numbers of rail victims across Europe over the last decade which is a result of various safety measures introduced and enforced by EU and the countries themselves within the time period described.

Figure 16 displays individual values for the sample countries for the parameter of annual number of victims. In addition, the European average with a trend line, as presented on Figure 15, has been included for easier comparisons. Although the trends for the five sampled countries are not displayed, as the figure would be difficult to read then, the values are linked with a line to show that an overall trend is negative indeed and that the number of victims is decreasing on average, although occasional peaks do happen (e.g. in 2011 for Bulgaria or 2009 and 2010 for Italy). Interestingly, Sweden's trend line is rather flat and stays at the level of 39 victims on average annually, which is second lowest results out of the five. In contrast, the results for Bulgaria show two peaks in 2006 and 2011 when around 120 people were rail victims in each of these years. Moreover, despite relatively short length of track and small annual numbers of passenger-kilometres in Bulgaria (displayed on Figure 13), when compared with the United Kingdom's rail network, numbers of victims are still at a quite high level. 


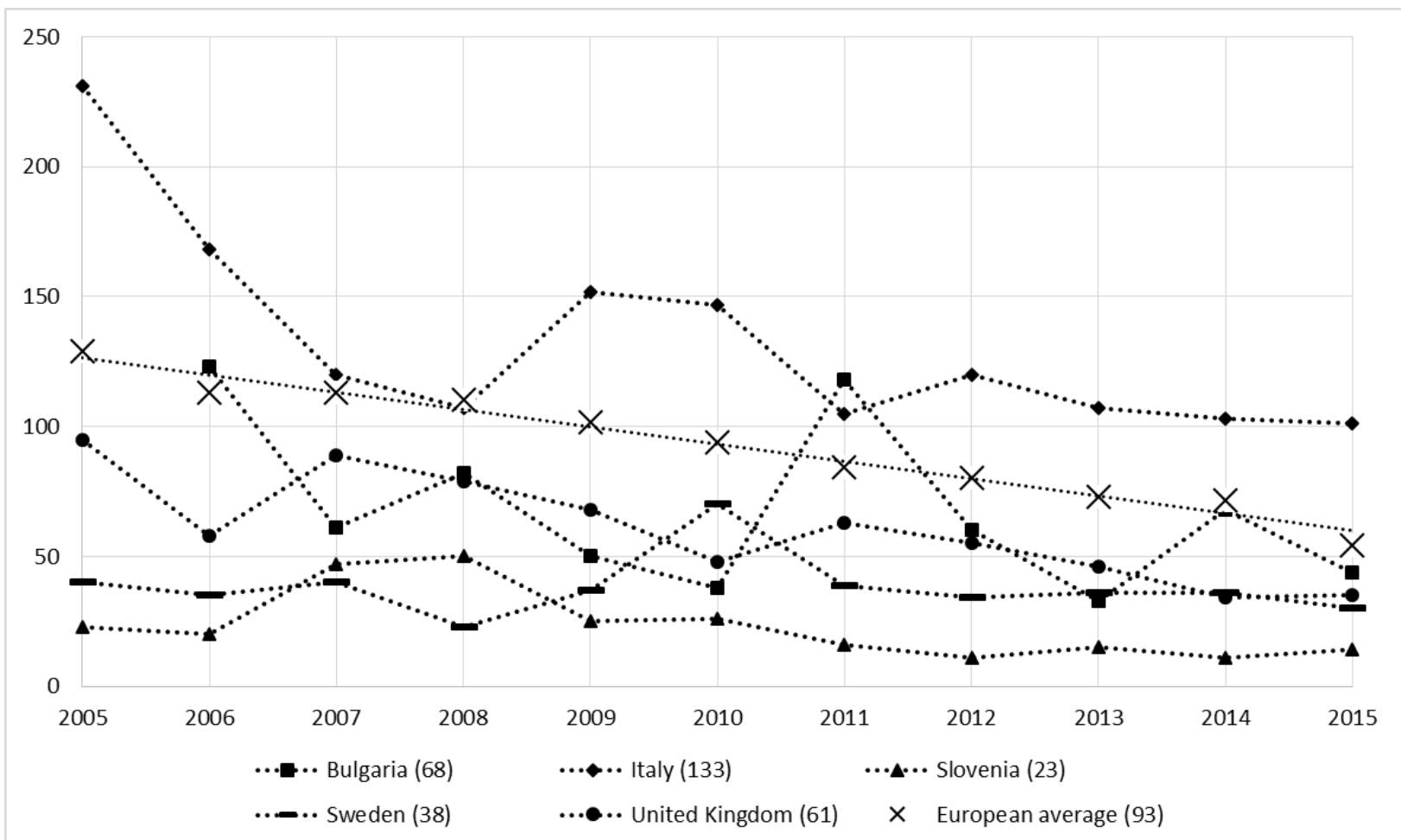

Figure 16. Annual number of rail victims against time for the sample countries [count]. Data source: Eurostat (2016)

Results displayed on Figure 17, with a distinction between killed and injured victims, shed some light on the numbers of rail victims in the five selected countries. It is clearly seen that Italy is leading the group with the highest numbers of killed and injured victims. Slovenia is at the other end of the spectrum with very few victims which on average stay at the level of under 20 injured and under 20 killed victims a year. Obviously there is a greater chance for an accident to occur in a more heavily utilised, larger system like the Italian, than in a smaller and less intense Slovenian railway system. However, Bulgaria with its medium-size railway system experienced many peaks, with up to 70 killed and up to 50 injured victims, between 2005 and 2015, meaning that rail accidents do occur in this country. In the United Kingdom the numbers have been decreasing and are currently at the level of about 30 killed and 10 injured victims per year, which are at the level similar to Sweden. Slovenia is the only country in the sample which managed to keep the number of killed victims below the number of injured for most of the period studied. This results also shows that in the sampled countries, unfortunately, there are more people killed than injured in rail accidents. 


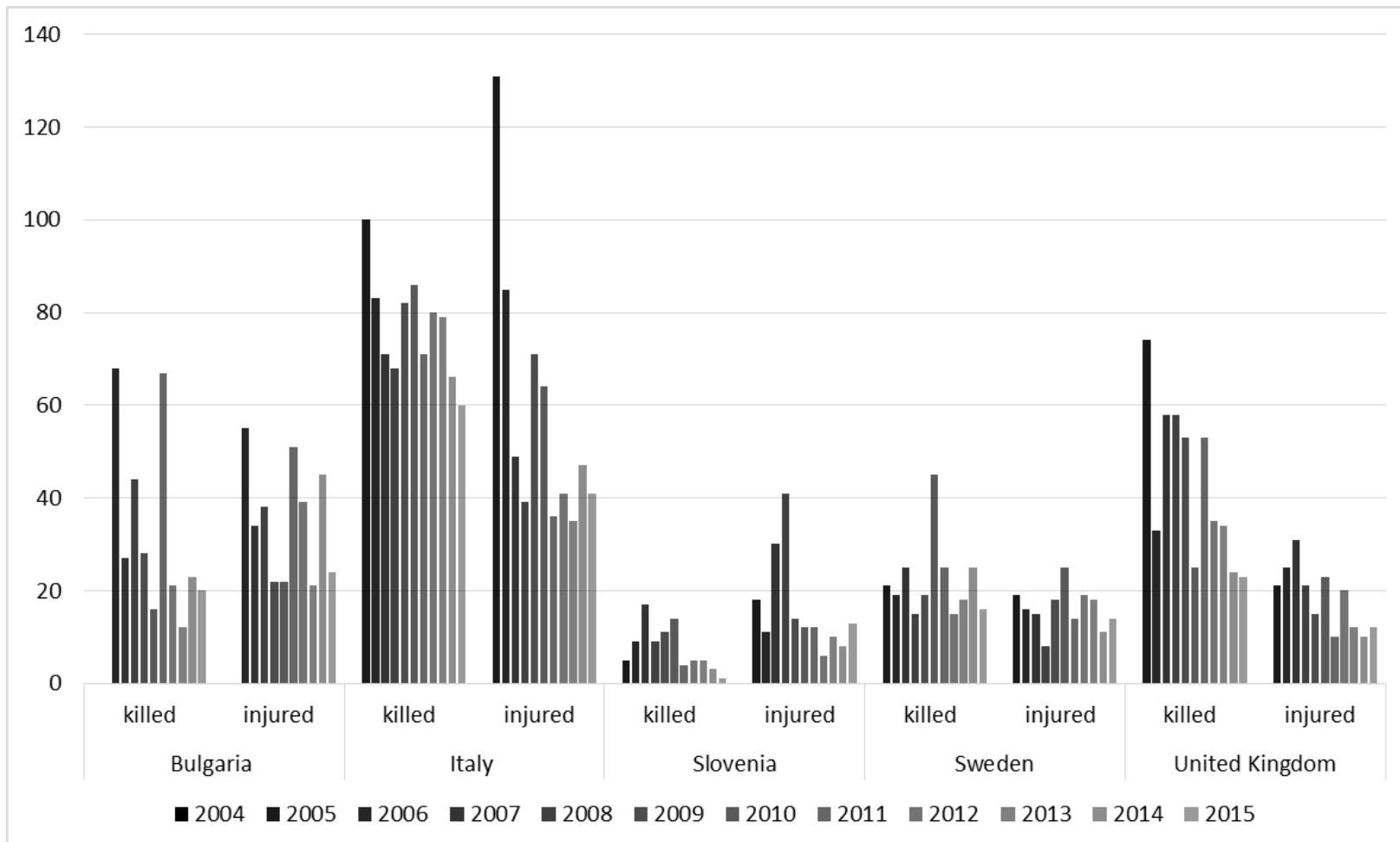

Figure 17. Annual number of rail victims by type of accident for the sample countries [count]. Data source: Eurostat (2016)

3.5 Capacity of railway vehicles in terms of seats and berths

A set of data on the capacity of railway vehicles in terms of seats and berths has been collected from Eurostat (2016). The data included numbers for a period of eleven years from 2003 to 2013. Figure 18 shows the plot of the average capacity of vehicles in Europe measured in 1000s of seats.

Values for years 2006 and 2012, were clearly identified as two outliers, which were misleading evaluation of the data. Removing the outliers, which are caused by an error in the data set, gave the results displayed on Figure 18, which shows a clear trend with the points closer to the regression line, less scatter. The figure shows a clear negative trend in the data; as time increases, average capacity of railway vehicles in Europe decreases. However, it must be stated that the quality of data for this KPI is problematic as there are many missing values for various countries (e.g. no data for the United Kingdom at all) or for some years only (e.g. missing values for Bulgaria for years 2005-2008 and 2013). Lack of consistency in the data does not give much confidence is assessing the results. 


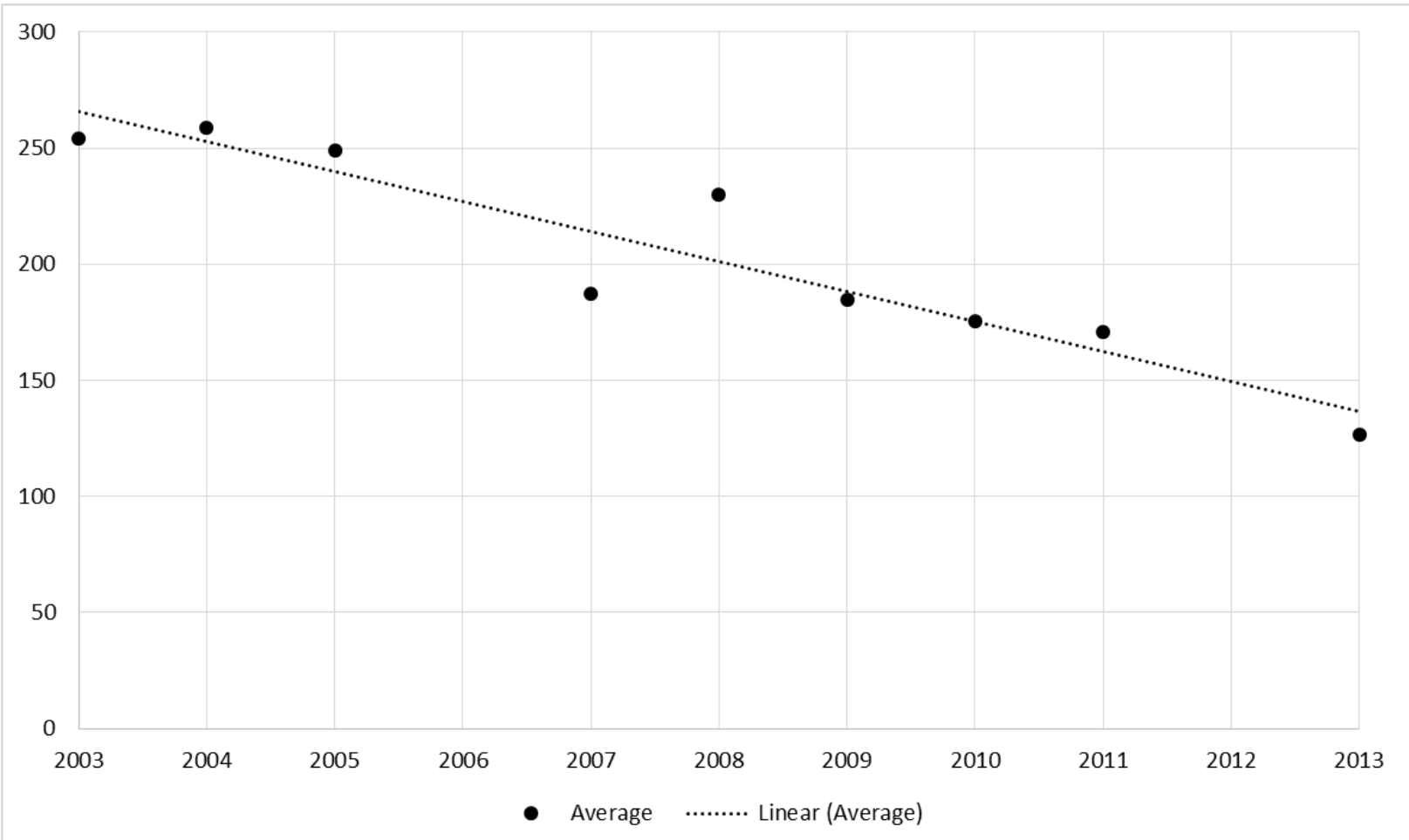

Figure 18. Average European capacity of passenger rail vehicles by category of seats and berths against time [1000 seats]. Data source: Eurostat (2016)

To further investigate the trends for capacity of passenger rail vehicles, data was collected on the expenditure on rolling stock in terms of investment to see if any trends in expenditure can be applied to the trends in the capacity of vehicles in terms of seats. Again, two clear outliers in the data in the year 2004 and 2011 were identified, due to error with the data.

Figure 19 with the clear outliers removed shows that there is a negative trend between the two variables; as time increases the expenditure on rolling stock decreases. This agrees with the figure showing the negative trend in seat capacity as it shows that investments are clearly not being made to deal with this decreasing trend in seat capacity. The decreasing trend in expenditure suggests that rail infrastructure managers are perhaps trying to reduce costs and are doing so by reducing the total capacity of passenger trains to further reduce maintenance costs.

The 2008 peak can be explained by massive investments in Hungary (301 million Euro) and Lithuania (329 million Euro). Overall the data set includes many missing values, also for the four out of the five countries sampled, for the period considered. In addition, it must be stated that Eurostat dictionary does not specify if investment expenditures on rolling stock, defined as: "expenditure for purchase of the new railway vehicles" (Eurostat, 2016b), relate to passenger vehicles only or include freight vehicles as well. 


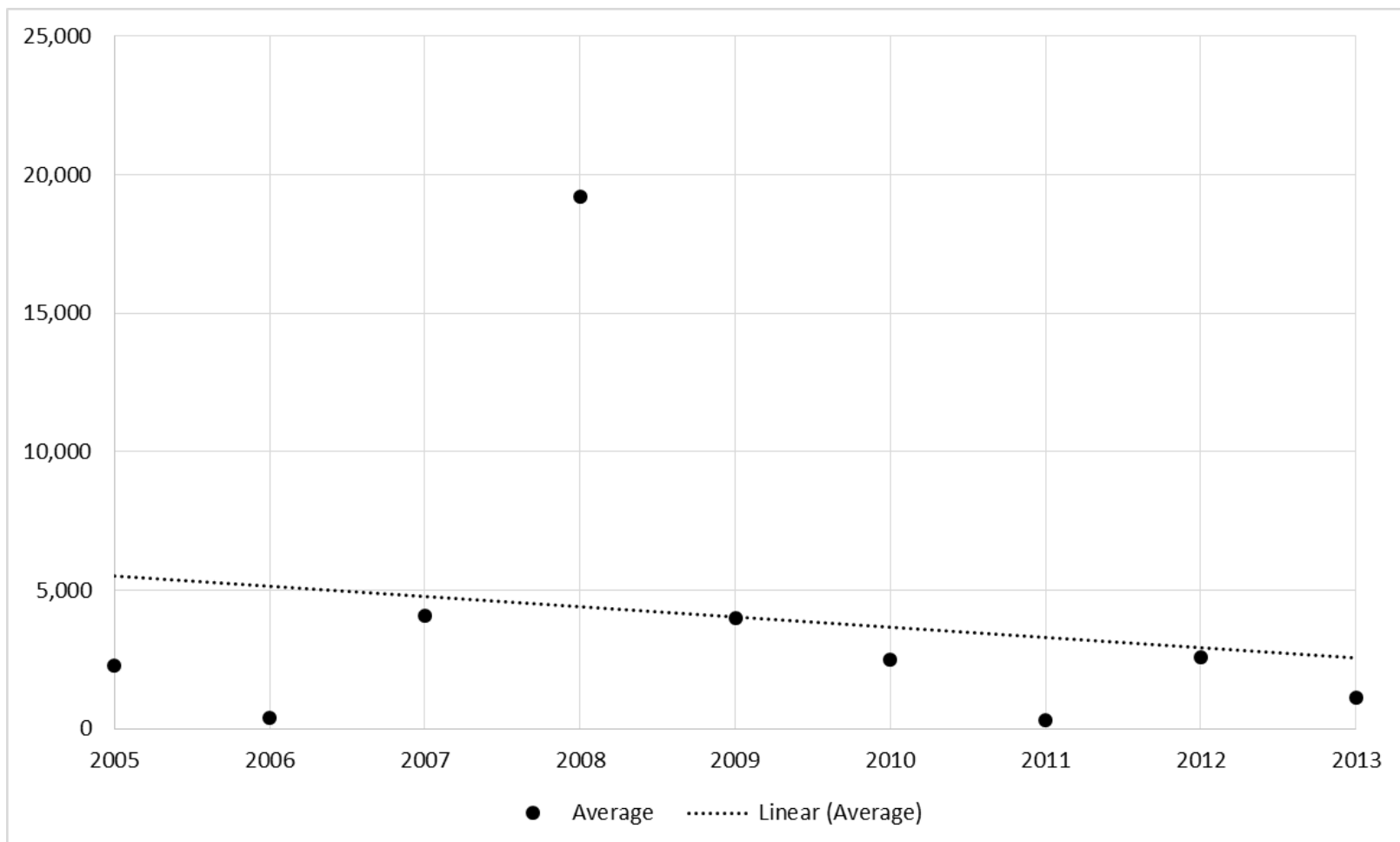

Figure 19. Average European expenditure on rolling stock investment against time [million Euro]. Data source: Eurostat (2016)

To further investigate situation in individual countries Figure 20 displays expenditure on rolling stock investment between 2005 and 2013. Trend lines have been replaced with point-to-point lines to highlight fluctuations in investments. It is well visible that the investment is kept at a similar level in all the sample countries with exceptions for 2005 for Slovenia (value of 4,771 million Euro) where number is very high showing large investments in the rolling stock in that year, possibly due to the fact that Slovenia joined EU in 2004 and started benefitting from large investments in rail infrastructure. However, this issue should be investigated with care as the number for 2004, not displayed on Figure 20, is also exceptionally high (value of 5,306 million Euro). Results for the United Kingdom and Italy, which investments are overall the highest out of the five countries stay at the level of between 1,000 and 2,000 [million Euro] for the years 2005 and 2009 to drop significantly from the year 2010 to below 500 [million Euro] per year. Although all the other countries experience peaks in various years, results for Sweden are over 500 [million Euro] between 2005 and 2007 to drop from 2008 to the bottom of the scale. Similarly, expenditure in Slovenia and Bulgaria is rather marginal when compared with the other countries selected, this is most probably due to a combination of economic reasons and decrease in the passenger use. 


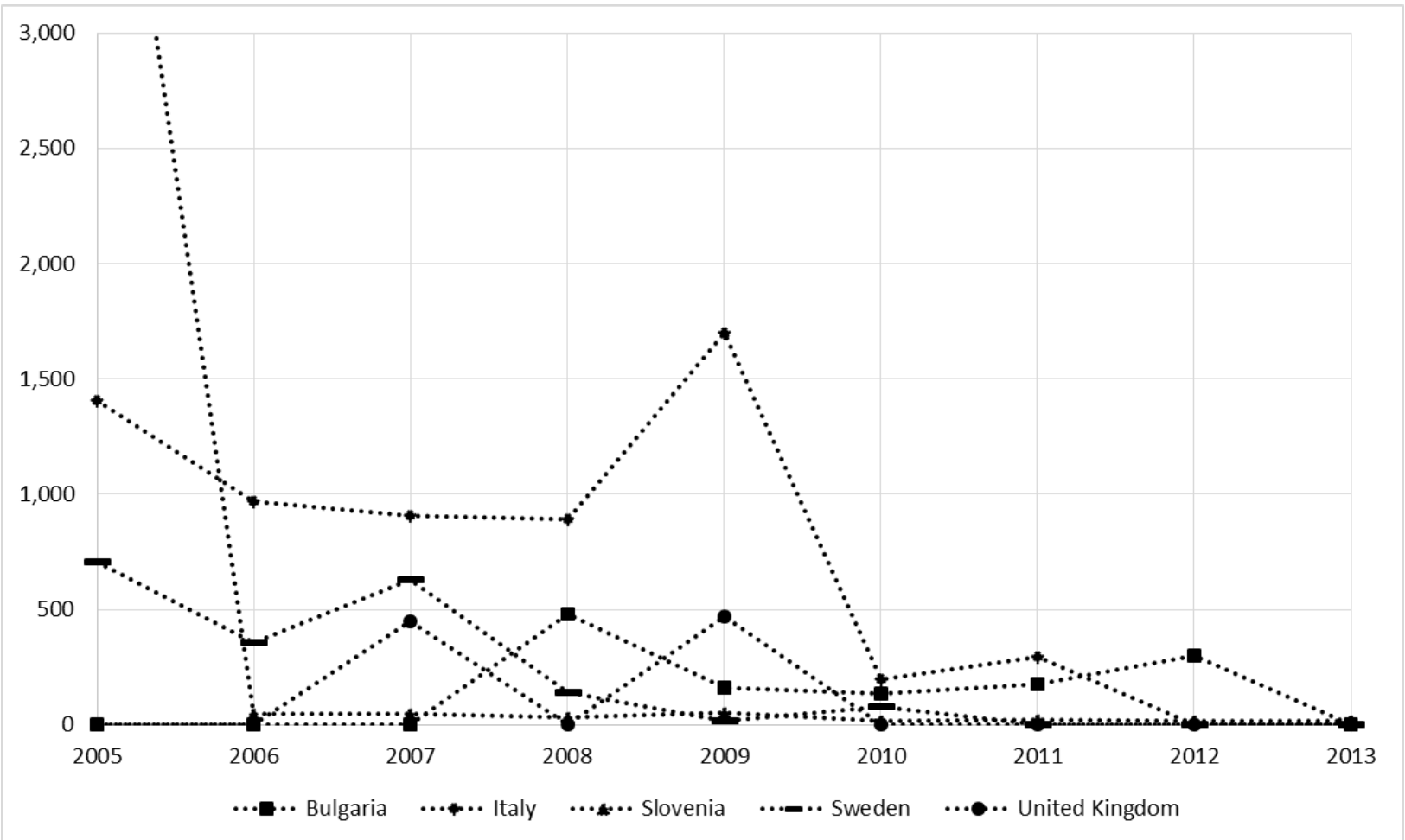

Figure 20. Expenditure on rolling stock investment against time per country for the sample countries [million Euro]. Data source: Eurostat (2016)

Figure 21 shows the trends for the sample countries in terms of capacity of rail vehicles expressed in the number of seats and berths. The graph only shows four out of the five sample countries as the United Kingdom had no data for the capacity of vehicles in this Eurostat set available. The figure shows that Sweden clearly has an increasing trend, the capacity of passenger vehicles increase from year to year, which reflects a steady increase in the annual passenger numbers, too (see Figure 13). The trend for Slovenia is slightly negative but is not as dramatic as Bulgaria's trend where in 2012 the numbers decreased to about 70. Apart from Sweden, the three other countries seem to fit the general European trend, which is rather negative in terms of number of rail seats and berths over the last decade. 


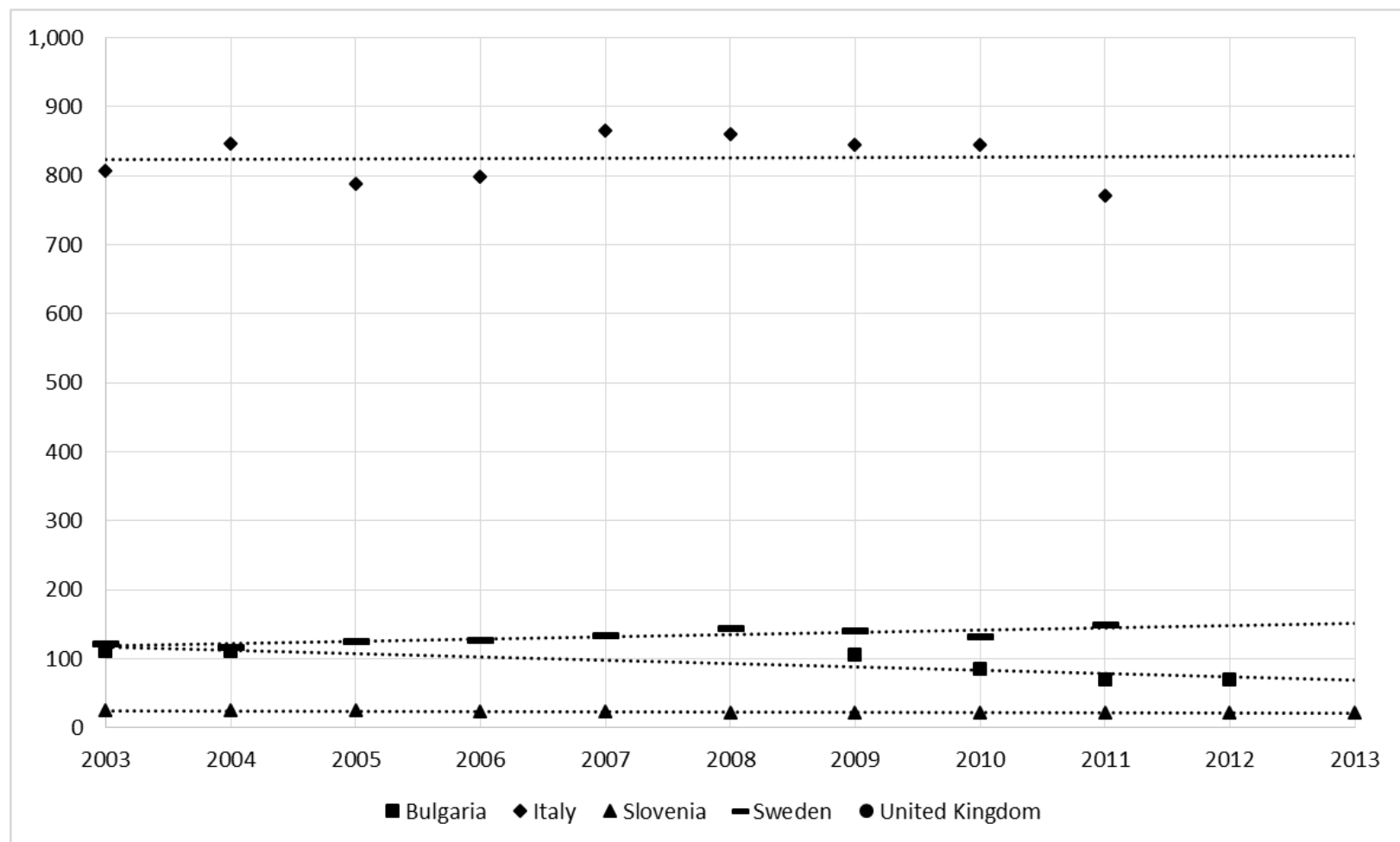

Figure 21. Capacity of passenger rail vehicles by category of seats and berths (sample) against time for the sample countries [1000 seats]. Data source: Eurostat (2016)

\section{Evaluation}

A set of rail-related data sources from Eurostat (2016) allowed conducting analyses of European rail system performance by taking into account a number of selected criteria. The first parameter to be discussed was the length of track and as seen on Figure 7 Europe has been experiencing a negative trend in this area for the last decade. This shows that the length of track is decreasing as time increases. Unfortunately, the data shows that instead of creating rail routes and laying new rail track to improve the overall accessibility and performance of the rail system, rail infrastructure mangers across Europe are generally shutting down/reducing the overall length of track (on average). Potentially fewer people will be able to use rail from more secluded/less popular areas reducing the overall accessibility of the rail systems. Therefore, this data provides evidence that the rail system in Europe is slowly shrinking.

Secondly, the graph for the annual number of passengers shows a positive trend, with the outliers within the data removed. This shows that the number of passengers is increasing as time increases, meaning that on average more people are using the rail systems within Europe. This larger increase is clearly not to do with increasing accessibility as the length of the rail systems is decreasing rather than increasing. Therefore, there must be other explanation for this positive trend, such as changes in lifestyle or an increasing demand for mobility to access education and employment. Sustainability issues may also play part in people's travel choices, but this avenue was not investigated in the paper.

Thirdly, the figure for the annual number of victims shows a strong negative trend; as the time increases, the number of victims decreases. This shows that rail safety has improved dramatically over the years and is continuing to improve. Therefore, this shows that in terms of rail safety, the rail systems in Europe are actually improving on average. However, in some countries railways are much safer (Sweden) mode of transport than in others (Bulgaria). 
Finally, the graph for the capacity of railway vehicles in terms of passenger seats shows a negative trend in the data once the two clear outliers had been removed; as time increases the average capacity of railway vehicles decreases. This is a worrying sign because, as discussed previously, the annual number of passengers is actually increasing. This shows that the rail operators are not taking into account the already stretched rail system and are actually reducing seat capacity rather than increasing the capacity of vehicles to accommodate for the increased number of passengers. Thus, this suggests that the rail system is not preparing for the increase in the number of passengers meaning that the performance of the rail system as a whole will suffer due to this lack of foresight. Therefore, this provides evidence that the rail system is not improving, as no significant changes influencing the trends can be seen in the data. However, there is a counter argument that the rail infrastructure managers may be reducing the capacity of vehicles but are increasing the number of trains on that route to accommodate for this change.

Comparing the trends for the length of track and capacity of rail vehicles it can be seen that both show a negative trend. The two variables maybe linked together as if the length of track is decreasing, infrastructure managers and train operators may try to reduce the capacity of vehicles as fewer rail routes are being used. So instead they are decreasing the capacity of vehicles to cope with this. This suggests that rail companies are focusing more on inner city/smaller scale networks as this may be more economically viable than increasing the number of rural track routes/less popular lines. Therefore, it seems as if the rail system is not helping those in more rural/less popular areas due to a lack of accessibility to the rail system i.e. the rail system is not performing as well as it could be as it is failing those who live in places that are not commonly passed through by popular commuter lines.

\section{Conclusions}

Drawing together all of the results presented in the paper, the data suggest that the rail system is not performing as well as it could be. The increase in passenger numbers is not being effectively dealt with by the rail infrastructure managers as instead of increasing the number of passenger seats to deal with this issue, they are actually reducing the number of vehicles with larger seat capacity. This is worrying as the system is already congested and this can only get worse with an increase in the number of passengers, as shown by Figure 13, if no improvements and investments are introduced. However, as discussed previously the managers of the rail systems may be increasing the number of trains on lines to accommodate with the decreasing capacity of rail vehicles, which is a positive move towards improvements.

Furthermore, rail operators at a European level are not expanding the system in order to improve accessibility for passengers in more rural and secluded areas. This would improve the quality of life for the individuals in these areas, allowing them to travel and exercise their mobility into towns and cities. This is an important issue as not only would it increase the quality of life for people living in secluded villages and towns, it could also potentially generate extra income for infrastructure managers as well as at journeys' destinations (employment, education, entertainment, etc.). However, the more pressing issue is that the railways are actually closing down areas of track. This may lead to many people been stranded with no mode of transport to access education or employment. Therefore, this indicates the level at which the rail system in Europe is performing is not very high. Moreover, it seems like the rail system is lagging behind the road network as the average length of motorways is increasing year on year.

Overall, despite some areas such as safety improving over time, the rail system seems to be underperforming in terms of total track length and removing higher capacity vehicles which will affect passenger numbers unless more a more frequent train timetable is being implemented. Unless specific and coordinated actions are taken to improve rail system performance in Europe, the system will be seemingly heading towards a critical limit.

\section{Recommendations}

To further investigate issues discussed in this paper, avenues for future research have been identified and are addressing the following issues.

Firstly, it is recommended to look into the extent that extending the length of track of a rail system affects the economy of the country and shapes the rail system itself in terms of sales and quality of service. This would give a more 
informed view on whether the rail infrastructure managers are trying to deal with the increase in passengers as discussed above. This research would benefit infrastructure managers and train operators, who could possibly investigate whether infrastructure and rolling stock investments would gain them more satisfied passengers using their services.

Secondly, it is proposed to look into the economics of the rail systems within Europe to investigate whether the rail infrastructure managers are reducing costs by decreasing capacity of passenger rail vehicles (reduce maintenance costs). The capacity issue is critical for the increasing demand for railway services in countries like the United Kingdom, therefore a research into the number of trains on lines where the capacity of vehicles is decreasing would be interesting. It could be investigated whether these two parameters affect one another and if they are somehow linked how this link affects the whole railway system.

Finally, an investigation which looks into more KPIs to be used in evaluating the performance of rail systems within Europe would allow for a more comprehensive overview of the railway systems across EU and comparisons of their performance from various perspectives.

\section{Additional Comments}

The outliers within the data occur due to the method used to calculate the average of the countries for each year. For example, a country with a large number of passengers may have no data for a few years (not contributing to the mean) but then suddenly the data is included. This leads to a large increase in the average for that year which can lead to outliers which give false impressions on the actual trends within the data.

\section{Acknowledgements}

Thomas Lamb was a Nuffield Research Placement holder who completed his placements with NewRail at Newcastle University in summer 2015. He worked on the initial version of the paper including original versions of the statistical analyses presented in this work.

\section{References}

BBC (2014) Rail regulator to investigate rail work delay chaos. Available at http://www.bbc.co.uk/news/uk-englandlondon-30607689 (Accessed 14/10/15)

CCTV (2016) China to build 30,000 kilometers high-speed railways by 2020. Available at http://english.cntv.cn/2016/01/12/VIDExvHxQhfEiwLpNyD5zQIX160112.shtml (Accessed 23/08/16)

Commission of the European Communities (2009) Communication from the Commission to the European Parliament and the Council on the production method of EU statistics: a vision for the next decade. Brussels, 10.8.2009, $\operatorname{COM}(2009) 404$ final. Available at

http://eur-lex.europa.eu/legal-content/EN/TXT/PDF/?uri=CELEX:52009DC0404\&from=EN (Accessed 17/09/2015) Department for Transport (2012) Britain to have new national high-speed rail network. Press release. Available at https://www.gov.uk/government/news/britain-to-have-new-national-high-speed-rail-network (Accessed on 23/08/16) Duranton, S., Audier, A., Hazan, J., Gauche, V. (2015) The 2015 European Railway Performance Index. Available at https://www.bcgperspectives.com/content/articles/transportation_travel_tourism_public_sector_european_railway_p erformance_index (Accessed September 2015)

Eurostat (2015) European rail statistics. Available at http://ec.europa.eu/eurostat/web/transport/data/database (Accessed 17/09/15)

Eurostat (2016) European rail statistics. Available at http://ec.europa.eu/eurostat/web/transport/data/database (Accessed 18/08/16)

Eurostat (2016b) RAMON - Reference And Management Of Nomenclatures. Available at http://ec.europa.eu/eurostat/ramon/foreword/index.cfm?targetUrl=DSP_FOREWORD (Accessed 22/08/16)

Kirchner, Ch. (2011) Rail Liberalisation Index 2011. Presentation delivered in Brussels on $20^{\text {th }}$ April 2011.

Available at https://goo.gl/CrCVOP (Accessed 16/08/16)

Parliament

(2015)

Meeting

future

transport

needs.

Available 
http://www.publications.parliament.uk/pa/cm201012/cmselect/cmtran/1185/118506.htm\#note74

(Accessed $15 / 10 / 15)$

Rail Technology Magazine (2015) Thousands claim for refunds after Clapham Junction chaos. Available at http://www.railtechnologymagazine.com/Inbox/thousands-claim-for-refunds-after-clapham-junction-chaos/105529 (Accessed 14/10/15)

Shift2Rail (2016) History of the initiative. Available at http://shift2rail.org/about-shift2rail/history-of-the-initiative (Accessed 23/08/16)

Telegraph (2013) British trains among best in Europe. Available at http://www.telegraph.co.uk/news/uknews/roadand-rail-transport/10528441/British-trains-among-best-in-Europe.html (Accessed 01/07/15)

The Guardian (2014) The 10 most crowded trains in the UK. Available at http://www.theguardian.com/uknews/2014/sep/10/10-most-crowded-uk-trains (Accessed 14/10/15)

UIC (2014) Railway Statistics 2013 synopsis. Available at http://www.uic.org/statistics (Accessed 14/10/15) 